\title{
El proceso de agriculturización en territorios con diferente matriz ecológico-productiva. El caso de la cuenca Mar Chiquita, provincia de Buenos Aires, Argentina ${ }^{1}$
}

\author{
Alejandra Auer², Néstor Maceira ${ }^{3}$ y Claudia Mikkelsen ${ }^{4}$
}

\begin{abstract}
RESUMEN
En las últimas décadas se profundizó el uso creciente y continuo de las tierras para cultivos agrícolas en la Región Pampeana Argentina, generando consecuencias en la dimensión ecológica, económico-productiva y socio-demográfica. El objetivo fue analizar el proceso de agriculturización en partidos de la Cuenca Mar Chiquita (Buenos Aires, Argentina) caracterizados por suelos con diferente aptitud de uso agrícola. La metodología se basó en analizar la variación de indicadores sobre variables que lo caracterizan agrupadas por dimensiones. Los resultados mostraron dos patrones del proceso de agriculturización, determinados por cambios en la dimensión socio-demográfica para los partidos agrícolas y con mayor afectación en la dimensión económico-productiva para los partidos ganaderos, los cuales mostraron un mayor impacto general del proceso y en la dimensión ecológica, aunque la diferencia en estos casos fue leve. Por tanto, se requiere una mirada integral de los procesos territoriales que evidencien su incidencia en la vulnerabilidad del sistema.
\end{abstract}

Palabras claves: Agriculturización, Matriz ecológico-productiva, Sustentabilidad.

\begin{abstract}
In recent decades, the increasing and continuous use of land for agricultural crops in the Pampean Region has been deepened, generating consequences in the ecological, economic-productive and socio-demographic dimension. The aim was to analyze the agriculturalisation process in Counties of Mar Chiquita Basin (Buenos Aires, Argentina) characterized by soils with different aptitude for agricultural use. The methodology was based on the analysis of the variation of indicators on the variables that characterize it grouped by dimensions. The results showed two different patterns of the agriculturisation process, mainly determined by changes in the variables of the socio-demographic dimension for agricultural Counties and with greater affectation of the economic-productive dimension for livestock Counties, which showed a greater overall impact of the process and the ecological dimension, although differences in these cases were slight. It requires a comprehensive view of the territorial processes that show their impact on the vulnerability of the system.
\end{abstract}

Key words: Agriculturisation, Ecological-productive matrix, Sustainability.

Artículo recibido el 15 de noviembre de 2017, aceptado el 20 de agosto de 2018 y corregido el 10 de septiembre de 2018. Consejo Nacional de Investigaciones Cientificas y Técnicas (CONICET) / Instituto Nacional de Tecnología Agropecuaria (Argentina). E-mail: aleauer@gmail.com

Instituto Nacional de Tecnología Agropecuaria (Argentina).E-mail: nmaceira@gmail.com

Consejo Nacional de Investigaciones Cientificas y Técnicas (CONICET) / Centro de Investigaciones Geográficas Socio-Ambientales y Centro de Investigaciones Geográficas (UNCPBA) (Argentina). E-mail: claudiamikkelsen@gmail.com 
En las últimas décadas se profundizó el uso creciente y continuo de las tierras para cultivos agrícolas en la Región Pampeana y otras zonas de Argentina. Este proceso definido como "agriculturización" (Barsky y Gelman, 2001; Paruelo et al., 2006; Navarrete y Gallopín, 2007; Zarrilli, 2010; Manzanal, 2017) es el resultado de una nueva lógica de acumulación conocida como el paradigma del "agribusiness" o "agronegocio" (Gras y Hernández, 2013). El agronegocio, se asienta sobre una matriz compuesta por un conjunto de innovaciones tecnológicas y gerenciales que promueve la producción a gran escala como estrategia para lograr mayor eficiencia productiva. Bajo este modelo, la superficie total cultivada en Argentina tuvo un crecimiento considerable, en coincidencia con la gran expansión del cultivo de soja. La producción de esta oleaginosa en el país pasó de 3,7 millones de toneladas en 1980/81 a 58,8 millones en 2015/16 (MAGyP, 2017). Esta situación generó la pérdida de bosques, pastizales y pasturas cultivadas, así como disminución en la diversidad de cultivos, lo que implica paisajes agrícolas más homogéneos y aumento en los costos ambientales de la agricultura (Paruelo et al., 2006; Aizen et al., 2009).

Entre los principales factores que contribuyeron al desarrollo de los cambios descritos se encuentran la flexibilización de las normas de arrendamiento (fines de la década de 1970) que permitió la generalización de los contratos por una cosecha, y la desregulación de la actividad agraria en 1991, la cual eliminó los organismos que regulaban la actividad (ej. Junta Nacional de Granos) (Teubal, 2009; Manzanal, 2017). Dichas medidas, sumadas a la ausencia de políticas de planificación del uso del suelo, otorgaron mayor peso en la toma de decisiones sobre el territorio a los grandes productores o empresas (Manuel-Navarrete y Gallopín, 2007). Los cambios de índole climática que venían ocurriendo (particularmente en la pluviosidad) (Teubal, 2009) incrementaron la realización de agricultura en zonas donde anteriormente no era posible, aumentando el valor de la tierra. Sin embargo, es con la liberación al mercado de la semilla de soja transgénica Round up Ready (RR) en 1996, la sostenida demanda de granos por los mercados externos y los cambios en los precios relativos de los productos agropecuarios cuando se instalaron compañías nacionales y multinacionales que orientaron sus estrategias hacia este sector, modificando la visión tradicional del productor agropecuario y los modos de vida de los actores locales (Teubal, 2009; Morello et al., 2006; Reboratti, 2006; Manzanal, 2017). Por tanto, se aprecia que los cambios en la esfera tecnológico-productiva influenciaron y fueron influenciados por cambios en las esferas económico-institucional, social y ambiental (Manuel-Navarrete y Gallopín, 2007).

Las características del proceso de agriculturización (PA) varían de acuerdo con los contextos ambientales y socio-económicos de cada región (Gasparri y Grau, 2006), determinando el tipo de consecuencias generadas y las diversas maneras de afrontarlas. En la Región Pampeana, la expansión de la superficie cultivada se produjo a expensas de tierras ocupadas por cultivos forrajeros perennes (Paruelo et al., 2006; Aizen et al., 2009) y de pastizales naturales (Herrera et al., 2009), reemplazando la rotación agrícola - ganadera tradicional por la agricultura continua. Este cambio provocó un desplazamiento de la ganadería hacia zonas marginales, así como su intensificación (terminación de los animales en corral o feedlots) (Paruelo et al., 2006; Teubal 2009). A su vez, las nuevas tecnologías permitieron avanzar sobre suelos bajos o someros, impactando en su conservación, dado que la sustentabilidad del proceso de agriculturización está ligada a su aptitud agrícola. A nivel socio-económico, generó prosperidad para algunos productores agropecuarios y ciertos sectores de las economías regionales que se beneficiaron con esta reactivación económica (Reboratti, 2006; Teubal, 2009). Se produjeron cambios en la estructura agraria y en los sistemas de producción y distribución (Reboratti, 2006), dado que "el efecto soja" (cuyo paquete 
tecnológico facilita, al simplificarlo, la producción en gran escala) favorece la concentración productiva y el arrendamiento de campos de terceros por parte de los grandes productores (Manzanal, 2017). El análisis del proceso de agriculturización requiere del análisis de los nuevos actores sociales que intervienen en la actividad, como los proveedores de agroquímicos, los contratistas y las grandes compañías exportadoras (Liberali, 2010; Craviotti 2005). Si bien los productores pampeanos fueron protagonistas de estas transformaciones empiezan a tomar mayor consciencia sobre el tema de la sostenibilidad, en parte porque saben que su actividad se realiza dentro de una sociedad que los evalúa (Manuel-Navarrete y Gallopín, 2007). Aun así, queda camino por recorrer en incorporar los costos sociales y ambientales de sus decisiones.

Los pequeños productores, en general con escasa capacidad de acceso al capital económico, optaron en gran parte por arrendar a terceros o vender sus explotaciones, llevando esto a una simplificación de la estructura social rural y generando un debilitamiento de las comunidades rurales locales, con desaparición de pequeños y medianos productores (Reboratti, 2006; Gras y Hernández, 2016). Según el Censo Nacional Agropecuario 2002, en la Provincia de Buenos Aires el tamaño de las explotaciones agropecuarias (EAPs) más grandes (más de 1000ha) aumentó un $20 \%$, mientras que la cantidad de EAPs pequeñas o medianas (hasta 200ha) disminuyó un 47\% entre 1988 y 2002 (INDEC, 2002). La pérdida del estrato de productores de menor tamaño se agudiza con la profundización del modelo agropecuario actual basado en la incorporación de tecnologías que dependen de mayores cantidades de capital invertido (Manzanal, 2017). De esta manera, el sector se convierte progresivamente en una "agricultura sin agricultores" (Domínguez y Sabino, 2006: 250), creciendo la agricultura de contrato y dejando de ser esta actividad el medio de vida para muchos productores, especialmente familiares (Teubal, 2009). Se transforma también el mercado laboral rural, resultando en el desplazamiento de los trabajadores menos calificados (asociados directamente a la producción), predominando empleos especializados en toda la actividad (Manuel-Navarrete y Gallopín, 2007; Teubal, 2009). A su vez, se evidencia una reducción considerable de la población rural y un crecimiento de las ciudades cabeceras, principalmente en las áreas más afectadas por la expansión de la soja (Reboratti, 2006).

Lo hasta aquí expuesto pone en evidencia transformaciones en el sistema socio-ecológico que afectan directamente al territorio rural, acentuando la inequidad en términos de bienestar de la comunidad local. La comunidad científica y algunas organizaciones no gubernamentales han alertado sobre los riesgos que implica este modelo en relación a la sustentabilidad de los sistemas ecológicos y sociales (Paruelo et al., 2006; Adámoli, 2006; Porto-Gonçalves, 2006; Manuel-Navarrete y Gallopín, 2007). Diversos estudios analizaron las consecuencias ambientales derivadas del modelo agrícola actual (e.g. Laterra et al., 2011; Jobbágy, 2011), que incluye la pérdida de biodiversidad nativa (Sabatino et al., 2010), erosión y pérdida de nutrientes del suelo (Adámoli, 2006; Aizen et al., 2009), degradación de la calidad del agua (Quirós et al., 2002), contaminación ambiental por agroquímicos (Aparicio et al., 2015) o la pérdida en la provisión de servicios ecosistémicos (Tapella, 2011; Barral y Maceira, 2012; Auer et al., 2017). Otros analizaron las consecuencias sociales del modelo, como el desplazamiento de la población rural y su cultura (Sili, 2005), la pobreza y migración hacia centros urbanos (Adámoli, 2006) o la desocupación, congestión urbana y polarización social (Morello et al., 2006). Sin embargo, son pocos los casos en la región que estudiaron el territorio considerando ambas dimensiones de manera integral (Tapella, 2011).

El territorio rural puede ser entendido como un sistema socio-ecológico donde interactúan el espacio biofísico natural y un complejo conjunto de actores sociales, el cual es asiento de acti- 
vidades productivas, relaciones sociales, fuente de identidad cultural y sentido de pertenencia (Brown \& Raymond, 2007; Llanos Hernández, 2013). Su estudio integral requiere contar con una mirada que tome en cuenta su complejidad -en lugar de intentar reducirla-, reconociendo las características y problemáticas locales como también su relación con territorios alejados pero que inciden en la dinámica local y en el conjunto de prácticas y decisiones que aplican los diversos actores. Es central en este tipo de estudios considerar el dinamismo propio del desarrollo sustentable, definido como la satisfacción de "las necesidades de la generación presente sin comprometer la capacidad de las generaciones futuras para satisfacer sus propias necesidades". (Comisión Internacional sobre Medio Ambiente y Desarrollo de las Naciones Unidas, 1987). Para ello, se requiere estudiar la tendencia actual y sus consecuencias, de forma de comprender los posibles escenarios futuros.

La construcción de indicadores para la evaluación de dicha sustentabilidad desde un abordaje que considere las dimensiones ambiental, social, económica e institucional (Reed et al., 2005), es entendido como un aporte central para la toma de decisiones. El marco conceptual adoptado para este trabajo es el del sistema de indicadores de desarrollo sostenible para Argentina (desarrollado por la CEPAL) cuyo objetivo es la mejora continua en la calidad de vida de la población (SAyDS, 2009). El uso de este marco conceptual permite una lectura integrada de los indicadores y sus interacciones y una visión holística de la sostenibilidad del desarrollo del sistema analizado, y constituye una herramienta para la fijación de políticas púbicas integradas (SAyDS, 2009). Sin embargo, para avanzar en este último punto es necesario incorporar en el análisis el entramado económico-institucional derivado del desarrollo histórico de las políticas públicas vinculadas a la temática y el dinamismo que caracteriza a este tipo de procesos territoriales (Manuel-Navarrete y Gallopín, 2007). Este dinamismo no sólo proviene de las innovaciones tecnológicas, sino también de la multiplicidad de actores sociales vinculados a la actividad que se incorporan, desaparecen y se reconvierten (Liberali, 2010).

El objetivo de este trabajo fue analizar el proceso de agriculturización (PA) a través de distintos indicadores agrupados bajo las dimensiones ecológica, económico-productiva y socio-demográfica, en partidos de la Cuenca Mar Chiquita (sudeste de la provincia de Buenos Aires) caracterizados por suelos con diferente aptitud de uso agrícola, lo cual condicionó históricamente la predominancia de sistemas productivos agrícolas, ganaderos o mixtos. Se tomó en cuenta el aspecto temporal con el fin de comprender la evolución de los indicadores y discutir los efectos futuros esperables de continuar la tendencia. La hipótesis planteada fue que el PA impacta de diferente manera sobre variables ambientales y socio-económicas según la matriz productiva predominante en cada territorio. La predicción fue que, en partidos predominantemente agrícolas, el PA impactaría principalmente en la dimensión social, mientras que, en partidos predominantemente ganaderos, el PA afectaría principalmente las dimensiones ecológica y económica. La lógica tras estas predicciones asume que en territorios caracterizados por tierras predominantemente agrícolas la agricultura ya habría avanzado sobre la mayor parte de los ecosistemas naturales y desplazado a otras actividades productivas, generando como consecuencia principal el despoblamiento rural. En cambio, en territorios donde la actividad principal fue históricamente la ganadería debido al predominio de tierras con escasa aptitud agrícola, la agricultura debería avanzar de manera más dispersa (utilizando aquellos pequeños parches con mayor aptitud), aumentando así la diversidad productiva, pero a expensas de la pérdida de ecosistemas naturales y sus servicios ecosistémicos. Evidencias de que el proceso de agriculturización en el sudeste bonaerense 
avanzó siguiendo el patrón descrito asociado a las características de los suelos que se presenta en Zelaya (2011), Lima et al. (2011) y Zelaya et al. (2016). Estos trabajos, no obstante, no analizan los aspectos socio-ecológicos y socio-económicos considerados en el presente estudio.

\section{Área de estudio}

El área de estudio fue la Cuenca Mar Chiquita (Figura $N^{\circ} 1$ ), ubicada en el sudeste de la provincia de Buenos Aires, cubre alrededor de un millón de hectáreas y puede considerarse representativa de los principales procesos de cambio de uso del suelo vinculados al avance e intensificación de la agricultura en la región, presentando además un importante valor cultural y turístico-recreativo. En la cuenca se encuentran dos grandes áreas ecológicas diferentes: un sector de sierras y tierras altas (pedemonte del sistema de Tandilia) correspondiente a la subregión de la Pampa Austral, cuyo uso es principalmente agrícola con predominio del cultivo extensivo de granos y presencia de explotaciones destinadas a la horticultura (cinturón hortícola de Mar del Plata), y otro sector de tierras bajas correspondiente a la Pampa Deprimida, cuyo uso predominante es ganadero, dedicado principalmente a la cría bovina (León, 1991). Dentro de esta última subregión se diferencia un tercer sector correspondiente a la zona costera y de la laguna Mar Chiquita, de alto valor turístico y para la conservación de la biodiversidad, declarada Reserva del programa EI Hombre y la Biosfera (Man and Biosphere - MAB) de la UNESCO en 1996 (Zelaya, 2011). La cuenca Mar Chiquita está integrada por 11 partidos (que incluye total o parcialmente) (Cuadro $N^{\circ} 1$ ).

Figura $\mathrm{N}^{\circ} 1$

Ubicación geográfica, división política, subregiones ecológicas y sierras, lagunas y cursos de agua de la Cuenca Mar Chiquita

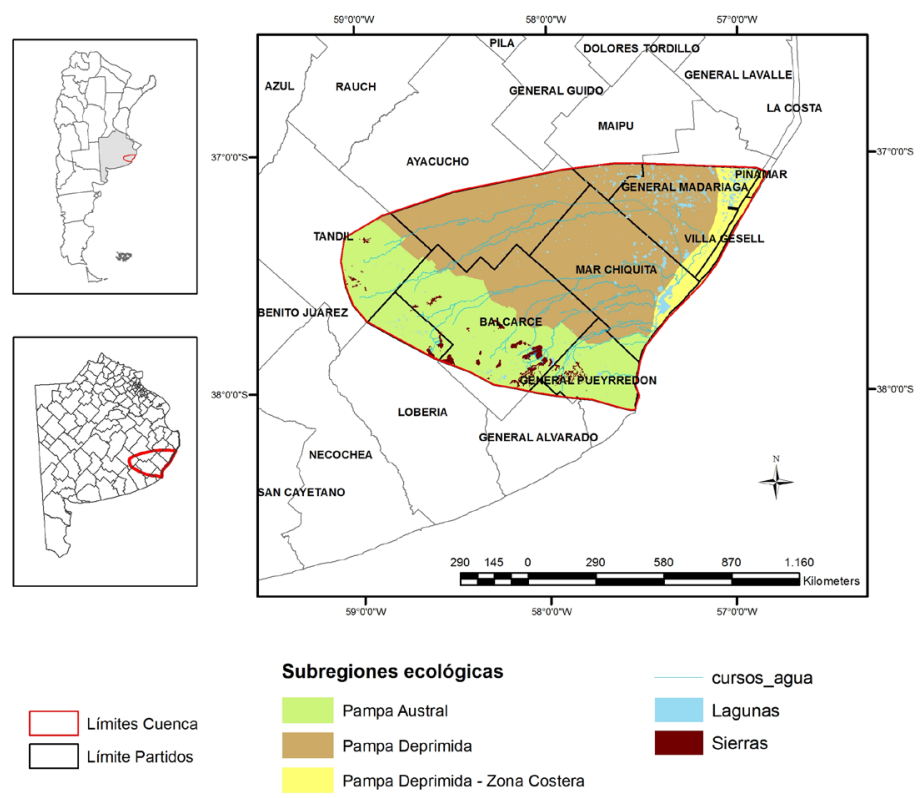

Fuente: Auer (2017). 
En su conjunto, estos partidos contribuyen al 10\% de la producción agrícola de la provincia de Buenos Aires, principalmente Lobería, Tandil y Balcarce (Campaña 2013/14; MAGyP, 2017). El incremento porcentual en los últimos 20 años (Campaña 2013/14 y 1993/94, MAGyP, 2017) en la producción agrícola (toneladas) de la Cuenca (168\%) fue mayor al experimentado por el total de la Provincia (133\%). Un comportamiento similar presentó el cultivo de soja durante ese período, cuya producción (toneladas) aumentó $2108 \%$ en los partidos de la Cuenca, mientras que el incremento en la provincia fue de $606 \%$.

En cuanto a la ganadería, los partidos del área estudiada proveen aproximadamente el $14 \%$ de las cabezas de ganado bovino provincial, con aportes principalmente de los partidos de Ayacucho, Tandil y Mar Chiquita (MAGyP, 2017). La disminución en la cantidad de cabezas de ganado bovino en los últimos 20 años (1992-2010) fue menor en los partidos de la Cuenca (-7\%) que en el total de la Provincia (-19\%) (MAGyP, 2017).

\section{Cuadro $\mathrm{N}^{\circ} 1$}

Partidos que integran la Cuenca Mar Chiquita, grado de inclusión y superficie dentro de la cuenca destinada a la agricultura y a la ganadería

\begin{tabular}{|c|c|c|c|c|c|c|c|c|}
\hline $\begin{array}{l}\text { Partidos que } \\
\text { integran la } \\
\text { Cuenca de Mar } \\
\text { Chiquita }\end{array}$ & $\begin{array}{c}\text { Superficie } \\
\text { del Partido } \\
(\mathrm{Ha})\end{array}$ & $\begin{array}{c}\text { Sup. } \\
\text { dentro de } \\
\text { la cuenca } \\
\text { (Ha) }\end{array}$ & \begin{tabular}{|c} 
Sup. \\
dentro de \\
la cuenca \\
(\%) (1)
\end{tabular} & $\begin{array}{c}\text { Sup. } \\
\text { coberturas } \\
\text { agrícolas }{ }^{4} \\
\text { (A) }\end{array}$ & \begin{tabular}{|c} 
Sup. \\
coberturas \\
ganaderas ${ }^{5}$ \\
(G)
\end{tabular} & $\begin{array}{c}\text { Sup. } A+G / \\
\text { Sup. } \\
\text { dentro de } \\
\text { la cuenca } \\
(\%)(2)\end{array}$ & $\begin{array}{l}\text { Sup. G / } \\
\text { Sup. A + } \\
\text { G (\%) (3) }\end{array}$ & $\begin{array}{l}\text { Tipo de } \\
\text { Partido }\end{array}$ \\
\hline Mar Chiquita & 312.934 & 312.934 & $100 \%$ & 81.953 & 205.080 & $92 \%$ & $71 \%$ & Ganadero \\
\hline Villa Gesell & 14.473 & 14.473 & $100 \%$ & 241 & 5.435 & $39 \%$ & & \\
\hline Balcarce & 414.683 & 373.220 & $90 \%$ & 188.409 & 166.941 & $95 \%$ & $47 \%$ & Agrícola \\
\hline G. Pueyrredon & 147.302 & 113.327 & $77 \%$ & 53.918 & 36.388 & $80 \%$ & $40 \%$ & Agrícola \\
\hline G. J. Madariaga & 304.801 & 212.912 & $70 \%$ & 47.824 & 141.698 & $89 \%$ & $75 \%$ & Ganadero \\
\hline Pinamar & 6.164 & 3.671 & $60 \%$ & 49 & 792 & $23 \%$ & & \\
\hline Ayacucho & 682.756 & 239.567 & $35 \%$ & 61.841 & 172.839 & $98 \%$ & $74 \%$ & Ganadero \\
\hline Tandil & 489.516 & 122.511 & $25 \%$ & 78.706 & 41.705 & $98 \%$ & $35 \%$ & Agrícola \\
\hline Maipú & 263.088 & 29.759 & $11 \%$ & 4.679 & 21.011 & $86 \%$ & & \\
\hline Lobería & 476.345 & 53.197 & $11 \%$ & 33.815 & 17.124 & $96 \%$ & & \\
\hline G. Alvarado & 162.934 & 4.720 & $3 \%$ & 4.242 & 1.057 & $112 \%$ & & \\
\hline Total & 3.274 .997 & 1.480 .291 & & & & & & \\
\hline
\end{tabular}

${ }^{4}$ Mapa coberturas, campaña 2013/14: cereales de invierno (con y sin soja de segunda), soja, otros cultivos, horticultura

${ }^{5}$ Mapa coberturas, campaña 2013/14: pasturas, pastizales, humedales.

Fuente: Elaboración propia.

Para este trabajo no se consideraron todos los partidos que integran la Cuenca, sino aquellos que para la campaña agrícola 2013-2014 cumplían los siguientes criterios: 1) superficie dentro de la Cuenca $>25 \%$, y 2) superficie dentro de la Cuenca destinada a la actividad agropecuaria > $50 \%$. Se consideraron partidos "ganaderos" a aquellos cuya superficie destinada a esta actividad 
era mayor al 50\% y partidos "agrícolas" al resto, si bien por ocupar en estos la ganadería entre el $30 \%$ y el $50 \%$ podría considerárselos mixtos con tendencia agrícola (Cuadro $N^{\circ} 1$ ). Los partidos seleccionados según este criterio fueron Mar Chiquita, Balcarce, General Pueyrredon, General Juan Madariaga, Ayacucho y Tandil.

\section{Metodología}

La metodología empleada está inspirada en el análisis de literatura especializada, principalmente en los trabajos de Gallopín (2006) sobre el uso de indicadores para el análisis del desarrollo sostenible en Honduras, Manuel-Navarrete y Gallopín (2007) donde se realiza la caracterización del síndrome de agriculturización utilizando el enfoque de síndromes de sostenibilidad del desarrollo, y Liberali (2010), Teubal (2009) y Reboratti (2006) donde se consideran las consecuencias sociales, económicas, ambientales y políticas de la expansión de la producción de soja en Argentina. Se adaptó el sistema de indicadores del marco del desarrollo sostenible para asegurar una mirada holística del proceso estudiado; no obstante, las variables consideradas responden a las características y efectos del PA, siendo por lo tanto indicadores del PA y no de sustentabilidad, al menos de manera directa. Concordamos también en que las variables incluidas en las diferentes dimensiones pueden en realidad estar asociadas a sus interrelaciones, como el caso de la variable "uso de insumos", incluida en la dimensión económica pero que podría ser considerada en la interrelación de ésta con la dimensión ecológica (SAyDS, 2009). Para cada dimensión se tomaron cuatro o cinco indicadores representativos del PA, que permitieron la comparación entre diferentes sitios (Cuadro $N^{\circ} 2$ ). Se los seleccionó procurando que abarquen la mayor complejidad posible, pero que a la vez sean simples y direccionalmente claros (Valentin y Spangenberg, 2000), que su medición sea objetiva y que los resultados puedan ser usados por diferentes tomadores de decisiones (Reed et al., 2005).

Para su selección y medición se consultaron fuentes bibliográficas y estadísticas oficiales, utilizando un enfoque descriptivo-comparativo. El estudio fue de tipo retrospectivo, donde se analizó el fenómeno longitudinalmente (descripción de la evolución de las variables en el tiempo) y transversalmente (comparación de dicha variación entre Partidos). La metodología no busca evaluar el valor absoluto de los indicadores, sino hacer un análisis diacrónico, midiendo la variación relativa de los indicadores en dos períodos (llamados "momentos", M1 y M2) (Gallopín, 2006). La fórmula empleada para su cálculo fue la siguiente:

$$
V I=\left(\left.I_{\text {final }}\right|_{\text {inicial }}\right) / I_{\text {inicial }}
$$

Donde "VI" es la variación del indicador, "I" es el valor del indicador al inicio (momento 1, M1) y al final (momento 2, M2). Los "momentos" (M1 y M2) varían entre algunos indicadores ya que dependen de la información disponible utilizada para su cálculo (ej. censo nacional agropecuario: años 1988 y 2002; censo poblacional: años 1991 y 2010; Cuadro № 2). En todos los casos los datos utilizados corresponden a cada partido, salvo la información referida a cobertura de suelos que corresponde a la superficie del partido que integra la Cuenca Mar Chiquita (Cuadro $N^{\circ} 1$ ). Dichos mapas diferencian las siguientes coberturas: Cereales de Invierno; Cereales de invierno 
con soja; Soja; Otros cultivos; Pastizales y pasturas; Forestal; Cuerpos de agua; Ciudades; Sierras; Horticultura; Dunas (Zelaya et al., 2016). Para la determinación de la variable superficie ganadera se procesaron los mapas de cobertura de las campañas 1999/2000 y 2013/14 (Zelaya et al., 2016) utilizando el programa ArcGis 9.3.

En la dimensión ecológica, los tres primeros indicadores del Cuadro $\mathrm{N}^{\circ} 2$ procuran determinar si hubo un aumento en la superficie agrícola total y del cultivo de soja en particular, y una disminución de la superficie ganadera (cobertura de pastizales y pasturas). La cobertura vegetal suele utilizarse como proxy de la provisión de servicios ecosistémicos y se reconoce que el reemplazo de la actividad ganadera extensiva por agricultura continua provoca una disminución de la biodiversidad y la provisión de varios servicios ecosistémicos claves (Barral y Maceira, 2012). La variación porcentual en el uso de la tierra agrícola en los principales cultivos es reconocida como información de base para la planificación y fijación de políticas relativas a la conservación y el ordenamiento territorial (SAyDS, 2009). Para estudiar si hubo una mayor intensificación ganadera, el indicador utilizado fue la cantidad de cabezas de ganado bovino dividida por la superficie ganadera ${ }^{5}$.

\section{Cuadro $\mathrm{N}^{\circ} 2$}

Dimensiones, Variables, Indicadores, Fuente de datos y años considerados para cada "momento" (M1 y M2), signo de cambio (SC) y factor de ponderación (FP)

\begin{tabular}{|c|c|c|c|c|c|}
\hline \multicolumn{2}{|r|}{ Dimensión - Variable } & Indicador utilizado (I) & Fuente utilizada & SC & FP \\
\hline \multirow{4}{*}{$\begin{array}{l}\frac{\pi}{0} \\
\frac{.0}{8} \\
\frac{.0}{0} \\
0 \\
\end{array}$} & Superficie cultivada & $\begin{array}{l}\text { Superficie sembrada } \\
(\mathrm{Ha})\end{array}$ & $\begin{array}{l}\text { SIIA - superficie sembrada } \\
\text { por partidos: promedios años } \\
\text { 1990-94 (M1) y 2009-13 (M2) }\end{array}$ & + & 3 \\
\hline & Superficie soja & $\begin{array}{l}\text { Superficie sembrada } \\
\text { con soja }(\mathrm{Ha})\end{array}$ & $\begin{array}{l}\text { SIIA - superficie sembrada } \\
\text { con soja por partidos: pro- } \\
\text { medios años 1990-94 (M1) y } \\
\text { 2009-13 (M2) }\end{array}$ & + & 3 \\
\hline & Superficie ganadera & $\begin{array}{l}\text { Superficie con pastiza- } \\
\text { les y pasturas }(\mathrm{Ha})\end{array}$ & $\begin{array}{l}\text { Mapas de coberturas por par- } \\
\text { tido: Campañas 1999/2000 } \\
\text { (M1) y 2013/14 (M2) - Mapas } \\
\text { base: Zelaya et al. (2016) }\end{array}$ & - & 3 \\
\hline & $\begin{array}{l}\text { Intensificación gana- } \\
\text { dera }\end{array}$ & $\begin{array}{l}\text { Cant. de bovinos (cab) / } \\
\text { sup. Ganad * sup. Den- } \\
\text { tro de la Cuenca }\end{array}$ & $\begin{array}{l}\text { SIIA - cabezas de ganado } \\
\text { por partidos: promedio años } \\
\text { 1992-97 (M1) y 2012-14 (M2); } \\
\text { Mapas coberturas por par- } \\
\text { tido: Campañas 1999/2000 } \\
\text { (M1) y 2013/14 (M2) }\end{array}$ & + & 2 \\
\hline
\end{tabular}

Dado que el dato de la superficie ganadera corresponde a la superficie del partido dentro de la Cuenca y la cantidad de cabezas de ganado bovinos corresponde al partido, este último dato se multiplicó por la proporción de la superficie del partido que integra la Cuenca. 
Continuación Cuadro $N^{\circ} 2$

\begin{tabular}{|c|c|c|c|c|c|}
\hline \multicolumn{2}{|r|}{ Dimensión - Variable } & Indicador utilizado (I) & Fuente utilizada & SC & FP \\
\hline \multirow{5}{*}{ 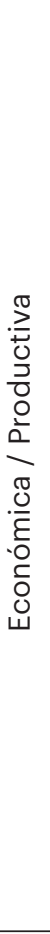 } & \multirow{2}{*}{$\begin{array}{l}\text { Uso de insumos en los } \\
\text { principales cultivos } \\
\text { (soja, girasol, maíz, } \\
\text { trigo) }\end{array}$} & $\begin{array}{l}\text { Insumos (miles litros) } \\
\text { utilizados }\left(^{*}\right) \text { en los prin- } \\
\text { cipales cultivos }\end{array}$ & $\begin{array}{l}\text { Insumos requeridos según } \\
\text { cultivos (Mosciaro et al., 2011); } \\
\text { SIIA - rendimientos de cultivos } \\
\text { por partidos: promedio años } \\
\text { 1990-94 (M1) y 2009-13 (M2) }\end{array}$ & + & 2 \\
\hline & & $\begin{array}{l}\text { Insumos (miles kilogra- } \\
\text { mos) utilizados }\left(^{*}\right) \text { en los } \\
\text { principales cultivos }\end{array}$ & $\begin{array}{l}\text { Insumos requeridos según } \\
\text { cultivos (Mosciaro et al., 2011); } \\
\text { SIIA - rendimientos de cultivos } \\
\text { por partidos: promedio años } \\
\text { 1990-94 (M1) y 2009-13 (M2) }\end{array}$ & + & 2 \\
\hline & $\begin{array}{l}\text { Concentración de las } \\
\text { principales activida- } \\
\text { des productivas }\end{array}$ & $\begin{array}{l}\text { Productor bruto geo- } \\
\text { gráfico (PBG) de las } 3 \\
\text { actividades principales } \\
\text { / PBG Total }\end{array}$ & $\begin{array}{l}\text { Dirección Provincial de Esta- } \\
\text { dística, Prov. de Buenos Aires } \\
\text { Año } 1993 \text { (M1) y } 2003 \text { (M2) }\end{array}$ & + & 3 \\
\hline & $\begin{array}{l}\text { Dependencia de la ac- } \\
\text { tividad agropecuaria }\end{array}$ & $\begin{array}{l}\text { PBG Agropecuario / } \\
\text { PBG Total }\end{array}$ & $\begin{array}{l}\text { Dirección Provincial de Es- } \\
\text { tadística, Prov. Buenos Aires } \\
\text { Año } 1993 \text { (M1) y } 2003 \text { (M2) }\end{array}$ & + & 3 \\
\hline & Precio de la tierra & $\begin{array}{l}\text { Valor de la Hectárea } \\
\text { (USD/Ha) }\end{array}$ & $\begin{array}{l}\text { Compañía Argentina de Tie- } \\
\text { rras: Año } 2003 \text { (M1) y } 2015 \\
\text { (M2) }\end{array}$ & + & 2 \\
\hline \multirow{5}{*}{ 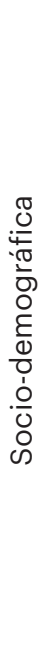 } & $\begin{array}{l}\text { Cantidad total de } \\
\text { EAPs }\end{array}$ & $\begin{array}{l}\text { Cantidad de EAPs tota- } \\
\text { les }\end{array}$ & $\begin{array}{l}\text { INDEC, CNA } 1988 \text { (M1) y } 2002 \\
(\mathrm{M} 2)\end{array}$ & - & 3 \\
\hline & $\begin{array}{l}\text { Cantidad de EAPs } \\
\text { de menor tamaño } \\
(<200 \mathrm{Ha})\end{array}$ & $\begin{array}{l}\text { Cantidad de EAPs < } \\
200 \mathrm{Ha}\end{array}$ & $\begin{array}{l}\text { INDEC, CNA } 1988 \text { (M1) y } 2002 \\
\text { (M2) }\end{array}$ & & 3 \\
\hline & $\begin{array}{l}\text { Tamaño medio de las } \\
\text { EAPs de mayor tama- } \\
\text { ño }(>500 \mathrm{Ha})\end{array}$ & $\begin{array}{l}\text { Tamaño EAPs > 50OHa } \\
\text { / cantidad de EAPs > } \\
500 \mathrm{Ha}\end{array}$ & $\begin{array}{l}\text { INDEC, CNA } 1988 \text { (M1) y } 2002 \\
\text { (M2) }\end{array}$ & + & 3 \\
\hline & $\begin{array}{l}\text { Población rural dis- } \\
\text { persa }\end{array}$ & $\begin{array}{l}\text { Cant. de habitantes en } \\
\text { zonas rurales no agru- } \\
\text { padas }\end{array}$ & $\begin{array}{l}\text { INDEC: Censo población. } \\
\text { Zona rural dispersa: } 1991 \text { (M1) } \\
\text { y } 2010 \text { (M2) }\end{array}$ & - & 3 \\
\hline & Población urbana & $\begin{array}{l}\text { Cant. de habitantes } \\
\text { en centros urbanos } \\
(>2000 \text { hab.) }\end{array}$ & $\begin{array}{l}\text { INDEC: censo población. Zona } \\
\text { urbana: } 1991 \text { (M1) y } 2010 \text { (M2) }\end{array}$ & + & 1 \\
\hline
\end{tabular}

$\left(^{*}\right)$ insumos utilizados en (barbechos químicos, implantación, fertilización, control de malezas y de insectos y enfermedades). Fuente: Elaboración propia.

En la dimensión económico-productiva se analizó si hubo un aumento en la utilización de insumos para la actividad agrícola como proxy de los cambios en las prácticas productivas. Se tomó como indicador el uso de insumos químicos para la realización de barbechos y para el control de malezas, insectos y enfermedades (en miles de litros) y para la implantación y fertilización de cultivos (en miles de kilogramos) (Mosciaro et al., 2011) considerando la superficie sembrada de los cuatro 
cultivos principales (soja, girasol, maíz y trigo) ${ }^{6}$. Para analizar si existió una concentración de las actividades productivas, dado que las actividades agropecuarias pasaron a estar dentro de las principales actividades económicas (verificado esto para todos los partidos salvo para General Pueyrredon), el indicador empleado fue la suma del producto bruto geográfico (PBG) de las tres actividades principales del partido dividida por el PBG total. En cambio, para analizar si hubo un aumento en la dependencia de la actividad agropecuaria se calculó el PBG agropecuario dividido por el PBG total. El último indicador de esta dimensión muestra si hubo un aumento en el precio de la tierra (USD/ha).

En la dimensión socio-demográfica se analizó si existió una disminución de la cantidad total de EAPs y específicamente de las EAPs de menor tamaño $(<200 \mathrm{Ha})$, y también si hubo un aumento en el tamaño medio de las EAPs de mayor tamaño $(>500 \mathrm{Ha}$ ), calculándose como el cociente entre la superficie total de las EAPs $>500 \mathrm{Ha}$ y la cantidad de dichas EAPs ${ }^{7}$. Por último, se analizó si hubo una disminución de la población rural dispersa (cantidad de habitantes no agrupados en zonas rurales) y un aumento en la población urbana (cantidad de habitantes en centros urbanos con más de 2000 hab.). Si bien el despoblamiento rural se dio tanto en la población rural agrupada (menos de 2000 hab.) como en la dispersa, se consideró sólo esta última dado que en el área de estudio hay poblaciones agrupadas que han tenido variaciones debido a temas no relacionados al PA, como las costeras y turístico-recreacionales (ej. Mar de Cobo).

Los indicadores de las variables de las diferentes dimensiones que caracterizan al PA se integraron en un índice del proceso de agriculturización (IPA) para comparar la incidencia del PA (total y según dimensiones) en los diferentes partidos. Para ello se utilizó la siguiente fórmula:

$$
\mathrm{IPA}=\Sigma \mathrm{VI} * \mathrm{SC} * \mathrm{FP}
$$

Donde "VI" es la variación del indicador explicado más arriba, "SC" es el signo del cambio, el cual es positivo (o negativo) si el aumento (o la disminución) en el indicador corresponde a un aumento del PA, $y$ "FP" es el factor de ponderación, el cual toma valores de 1, 2 o 3 según la importancia dada del indicador como característica del PA, siendo 3 la mayor importancia.

En el caso del uso de insumos químicos para la actividad agrícola, dado que se utilizaron dos indicadores para una misma variable por estar indicados en litros y kilogramos, para el cálculo del IPA se hizo un promedio del IPA de ambos indicadores. El signo de cambio se consideró positivo cuando el aumento del indicador corresponde a un incremento en el PA (ej. uso de insumos químicos) y negativo en el caso contrario (ej. tamaño de la población rural) (Cuadro $N^{\circ} 2$ ). Los valores obtenidos de la multiplicación de la variación del indicador por el signo de cambio se han normalizado considerando los valores máximos de cada indicador y cero, de forma de expresar todos los indicadores en una escala homogénea donde el valor máximo de agriculturización sea 1.

Dado que no todas las variables consideradas tienen la misma importancia para medir el PA, la fórmula para el cálculo del IPA incluye un factor de ponderación (Cuadro $N^{\circ} 2$ ) según la importancia relativa atribuida a cada variable en el PA. Los valores varían entre 1 y 3 , siendo 3 el más importante. La ponderación de las variables suele ser utilizada para reflejar la diferente importancia de las mismas en

\footnotetext{
Dado que sólo se consiguieron datos sobre la cantidad de insumos utilizados en la actualidad, este dato se utilizó para calcular el indicador en ambos momentos, en función de la cantidad de hectáreas sembradas de cada cultivo en cada período (MAGyP, 2017). Cantidades por hectáreas tomadas de Mosciaro et al., 2011.

Dado que las categorías en el CNA son de EAPs de 500 ha a 1000 ha y de más de 1000 ha, primero se calculó el tamaño medio de las EAPs por categoría y posteriormente se calculó el promedio de ambos.
} 
un determinado proceso, si bien el cambio en los pesos asignados puede hacer cambiar los resultados (Reed et al., 2005). En este estudio, la asignación de dicho valor se realizó sobre la base de una consulta bibliográfica previa y el criterio experto, realizándose en el mes de abril de 2016 una encuesta a 12 informantes calificados de diferentes áreas (agronomía, economía y sociología) del INTA EEA-Balcarce, a quienes se les solicitó, entre otras cuestiones, que indiquen la importancia de las diferentes variables consideradas para la medición del PA. Si bien en esta consulta la variable relacionada con utilización de insumos en cultivos resultó 'muy importante', se le asignó un valor de ponderación 'medio' dado que este indicador guarda relación con la superficie sembrada en general y con la de soja en particular. Con criterio similar se procedió con la variable de población urbana, la cual fue valorada como de 'mediana importancia' por los expertos, pero asignada con un factor de ponderación 'bajo' debido a que, dadas las características de las ciudades del área de estudio (ej. Mar del Plata, Tandil), los cambios en este indicador pueden estar relacionados a otros factores además del PA. Con estos ajustes se obtuvo un mayor equilibrio en el peso de las diferentes dimensiones.

\section{Dinámica de las dimensiones relevadas}

Los resultados mostraron dos patrones diferentes del proceso de agriculturización (PA) para los grupos de partidos estudiados, determinados principalmente por cambios en las variables de la dimensión socio-demográfica en el caso de los partidos agrícolas y con mayor afectación de la dimensión económica-productiva en los partidos ganaderos, en coincidencia con la predicción planteada (Figura $\mathrm{N}^{\circ}$ 2). Los partidos ganaderos mostraron un mayor impacto general del PA y en la dimensión ecológica, aunque la diferencia fue leve (Cuadro $N^{\circ} 3$ ).

Figura $\mathrm{N}^{\circ} 2$

Variación entre períodos del valor del indicador (VI) por el signo de cambio (SC) normalizados para cada indicador para cada dimensión en partidos agrícolas (AGR) y ganaderos (GAN)

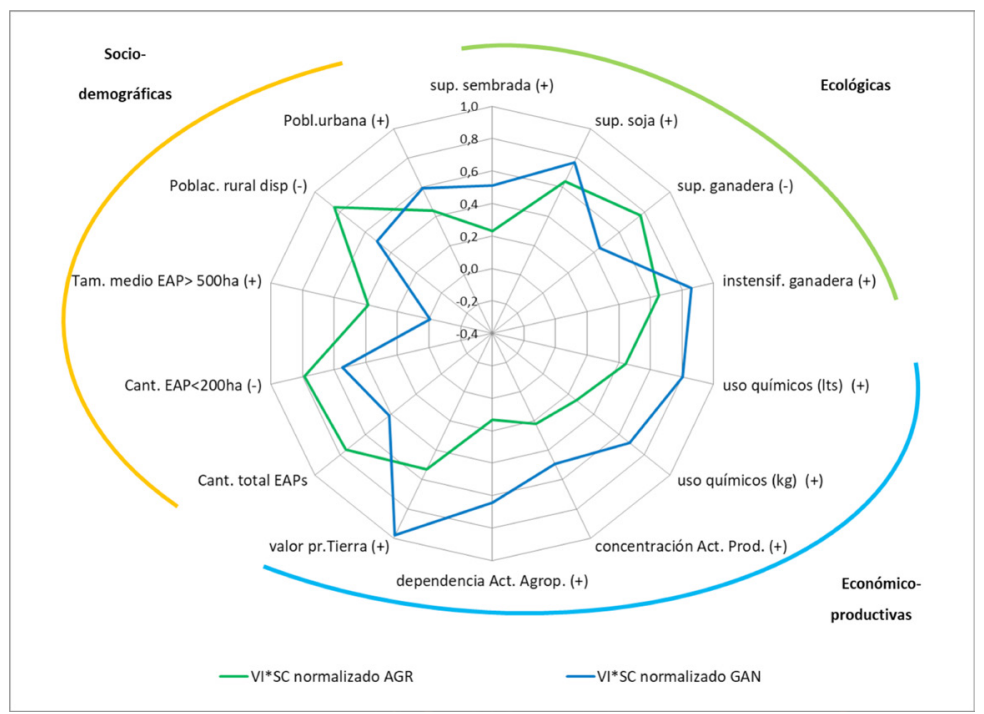

(+/-) Entre paréntesis al costado de cada indicador se muestra el signo de cambio esperado para el indicador ante un aumento en el proceso de agriculturización (PA) (positivo: a mayor PA, mayor variación positiva del indicador; negativo: a mayor PA, mayor variación negativa del indicador). Fuente: elaboración propia. 


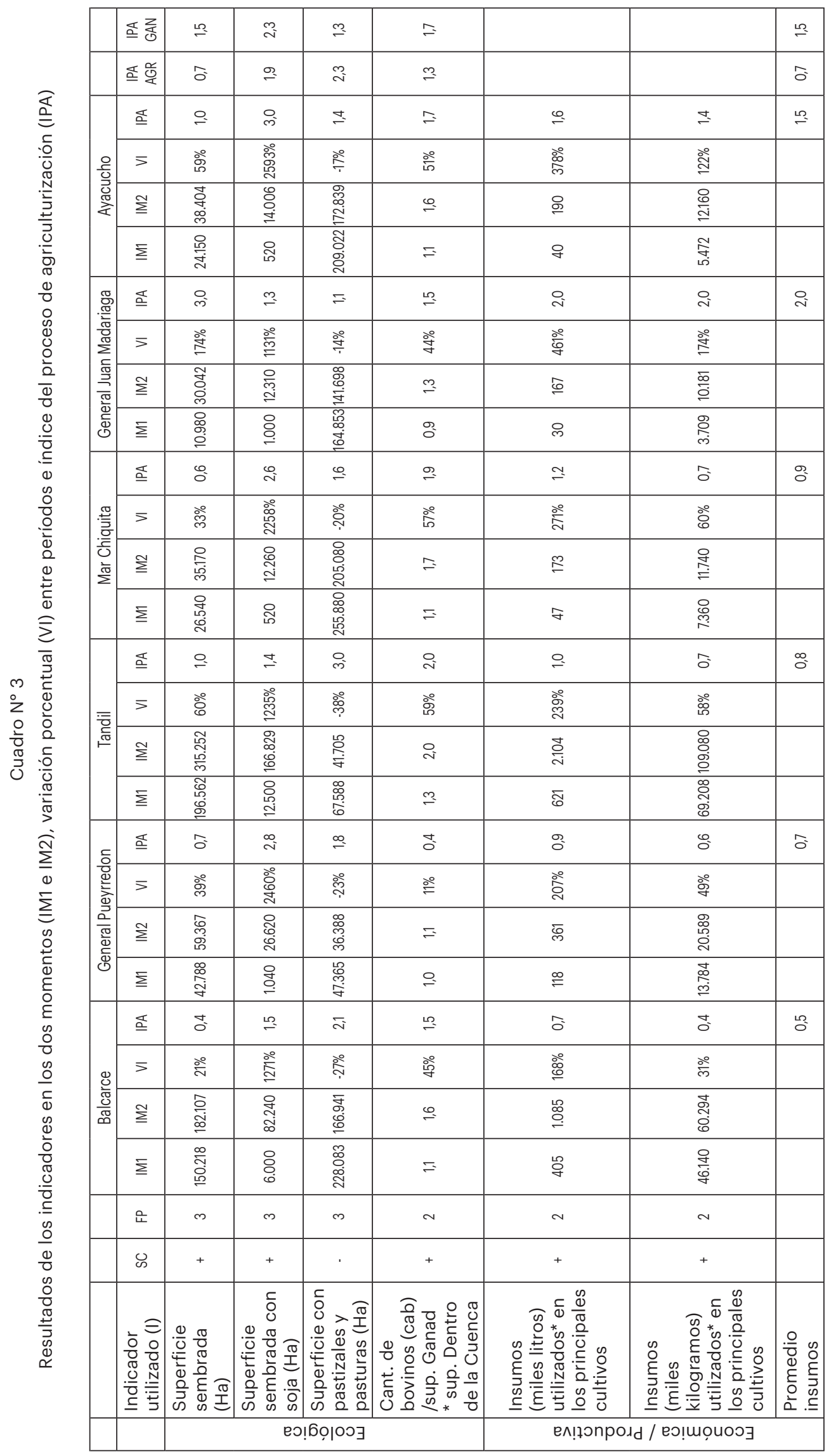




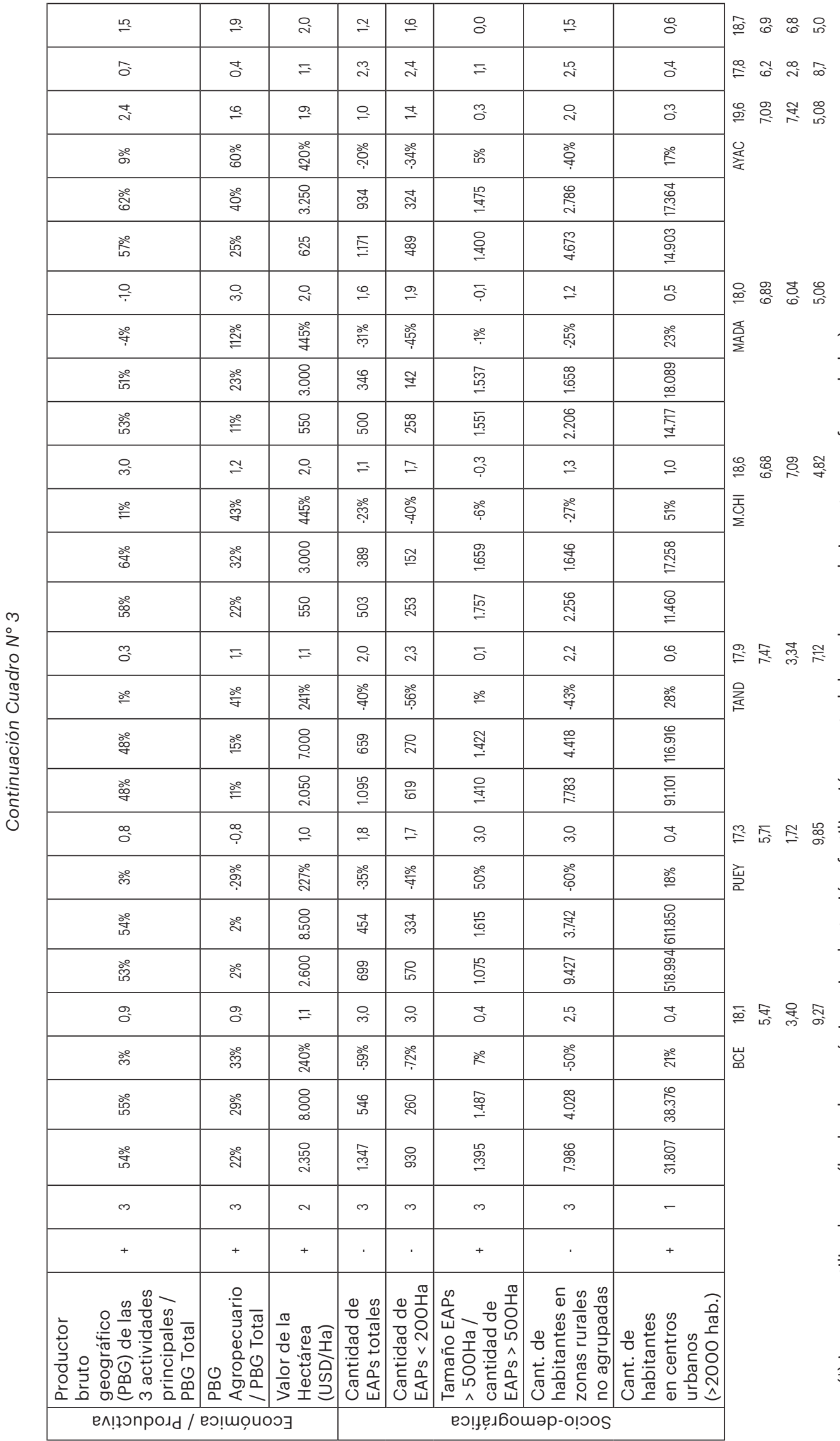




\section{Dimensión ecológica}

En esta dimensión, los partidos ganaderos mostraron un mayor impacto relativo en todos los indicadores, salvo en la reducción de la superficie ganadera (Figura $\mathrm{N}^{\circ} 2$; Cuadro $\mathrm{N}^{\circ} 3$ ). Si bien en términos absolutos la superficie sembrada y con soja fue considerablemente mayor en los partidos agrícolas (Figura $N^{\circ} 3$ ), la variación puede tener diferente significación en términos relativos según el contexto territorial, remarcando estos resultados la importancia de considerar las características propias del territorio bajo estudio. La proporción que representa el cultivo de soja dentro de la superficie sembrada pasó de un $4 \%$ en la década de 1990 (promedio años 1990-1994) en ambos grupos de partidos, a representar el 48\% alrededor del 2010 (promedio años 2009-2013) en los partidos agrícolas y el $37 \%$ en los partidos ganaderos. Esto se relaciona con la diversidad de cultivos anuales cosechados, la cual disminuyó en los partidos agrícolas que dejaron de producir lino, cebolla, ajo, mijo, alpiste y sorgo, aunque incorporaron cebada cervecera y colza. Los partidos ganaderos, por el contrario, aumentaron su diversidad de cultivos, ya que, si bien dejaron de producir lino, incorporaron la cebada cervecera, colza y alpiste (Figura $\left.N^{\circ} 4\right)$.

Figura $\mathrm{N}^{\circ} 3$

Resultados de los indicadores: a) superficie sembrada total y b) superficie sembrada con soja (hectáreas) en los dos períodos (M1 y M2) para los grupos de partidos (AGR; GAN)
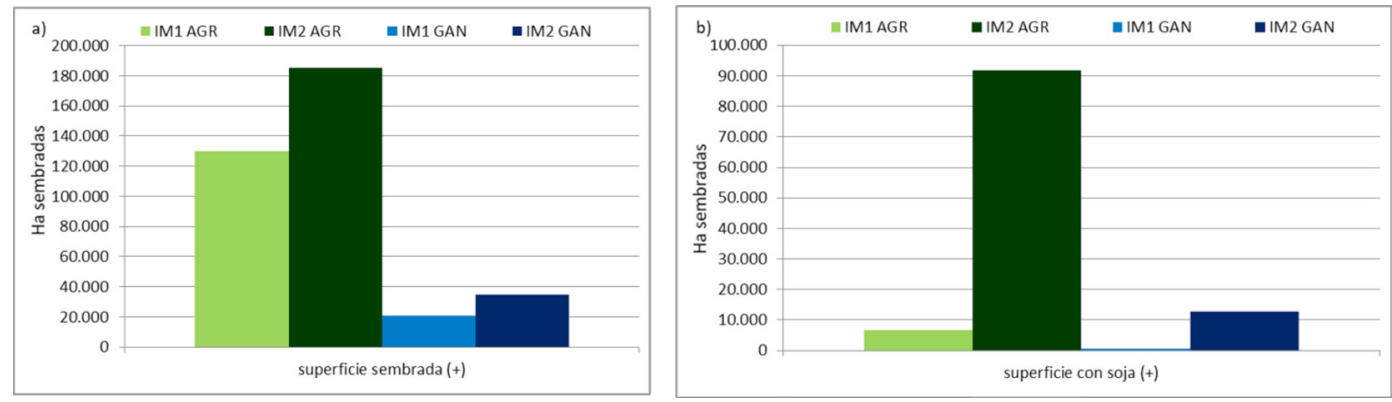

Fuente: Elaboración propia.

El avance de la agricultura sobre suelos de baja capacidad agrícola implica la posibilidad de degradación y consecuentemente la afectación en la provisión de servicios ecosistémicos (ej. amortiguación de inundaciones) por disminución de cobertura vegetal e infiltración (Jobbágy, 2011). Esta situación puede llevar a una mayor vulnerabilidad ecológica, derivada de la degradación de tierras, pérdida de biodiversidad y servicios ecosistémicos y contaminación del agua y del aire (FAO, 2007; Paruelo et al., 2006; Viglizzo, 2008) y a una mayor vulnerabilidad social, relacionada a los mayores índices de desempleo, pobreza y mala alimentación (Mikkelsen, 2008). Este es un aspecto importante a considerar, dado que, por ejemplo, en 2016, el $40 \%$ de los suelos de Balcarce con capacidad de uso no agrícola (capacidad de uso V a VIII) estaba cubierto con cultivos, representando el $11,5 \%$ del territorio del Partido (Angelini, 2017). De ese mismo informe surge que el $28 \%$ de los suelos con capacidad agrícola estaban con uso no agrícola (ej. urbanizaciones) siendo necesaria una planificación del uso del territorio de manera más eficiente y sustentable. Si bien en términos absolutos la disminución de la superficie destinada a la ganadería (pastizales y pasturas) fue levemente mayor en los partidos ganaderos, la variación relativa de este indicador fue mayor en los partidos agrícolas, donde disminuyó un 30\% entre períodos considerados (Figura $\mathrm{N}^{\circ} 4$ ). La relevancia de este resultado radica en la importancia que tienen los remanentes 
de pastizales naturales o semi-naturales como refugio de biodiversidad y como proveedores de servicios ecosistémicos en ambientes predominantemente agrícolas (Herrera et al., 2009). A su vez, el hecho que las mejores tierras se destinen a realizar una agricultura de cuasi monocultivo para exportación atenta contra la seguridad alimentaria local (Porto-Gonçalves, 2006).

Figura $\mathrm{N}^{\circ} 4$

Superficie sembrada (en hectáreas) de diferentes cultivos para dos períodos: promedio 1990-94 y promedio 2009-13, en los partidos considerados
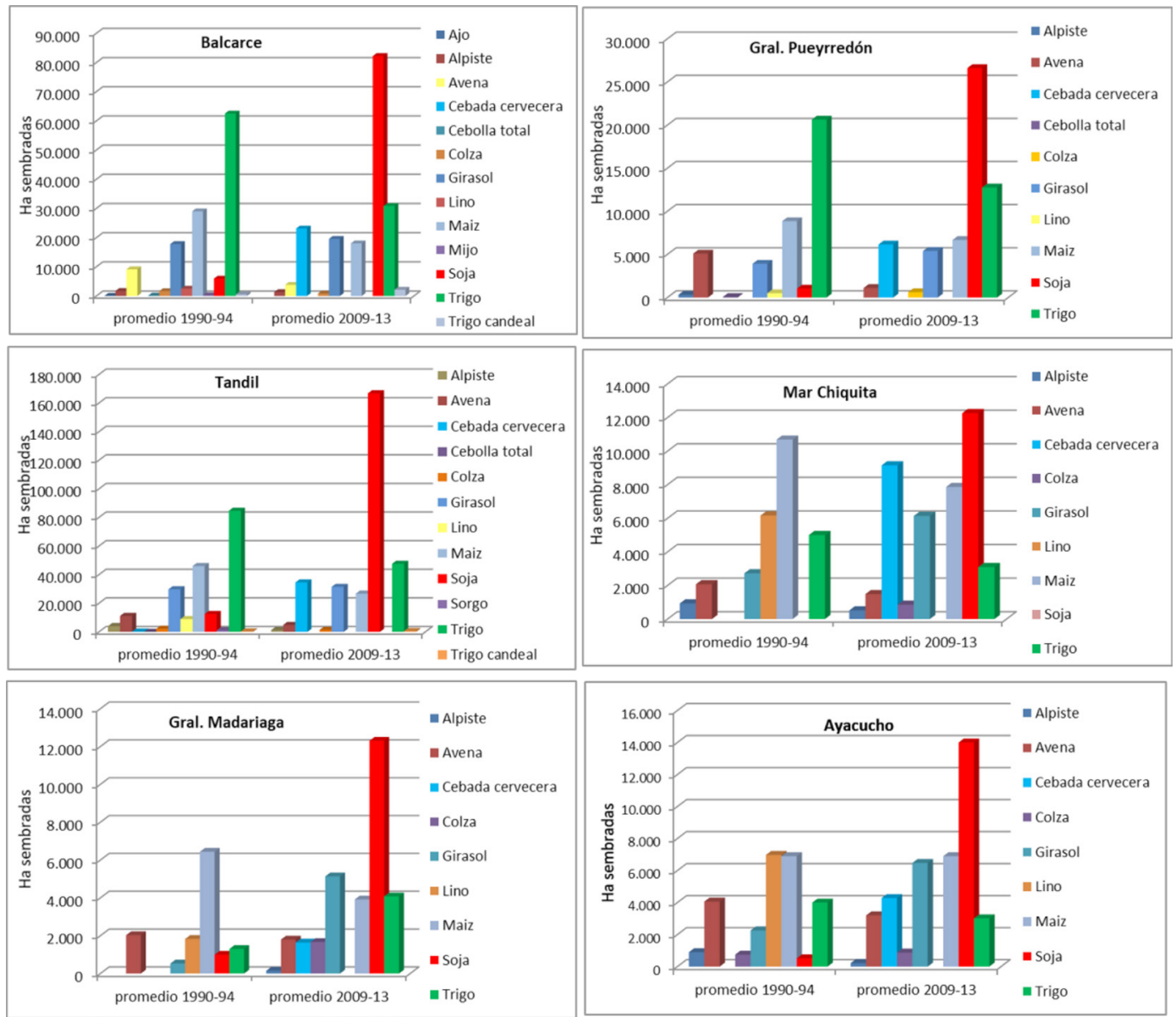

Fuente: Elaboración propia en información de cultivos sembrados base a, MAGyP (2017).

El indicador de intensificación ganadera aumentó en ambos grupos de partidos, aunque el incremento fue mayor en los partidos ganaderos (51\%), debido al aumento en la cantidad de cabezas de ganado (25\%) y la menor disminución de la superficie ganadera (-17\%) comparado con los partidos agrícolas donde, si bien esta reducción fue mayor (-29\%), hubo una leve baja en la cantidad de cabezas de ganado (-3\%) (Figura $\left.N^{\circ} 5\right)$. Aun así, la carga animal por unidad de superficie ganadera se mantuvo levemente mayor en los partidos agrícolas en ambos momentos. Estos resultados confirman la existencia de un proceso de intensificación ganadera paralelo al avance de la agricultura en la Cuenca Mar Chiquita (Zelaya, 2011). La práctica de engorde bovino a corral 
o feedlots como alternativa para obtener réditos de mercado ha crecido en la Región Pampeana en la última década (Moscuzza, 2010), aumentado la producción forrajera y la carga animal a través de la fertilización nitrogenada y la suplementación con maíz y/o concentrados. Este incremento de pequeños lotes sembrados (ej. con maíz) para ser utilizados como alimento para el ganado lleva a una retroalimentación positiva de aumento de intensificación ganadera y agrícola. La relevancia de este resultado radica en que la intensificación ganadera no sólo requiere de más insumos (salvo el recurso tierra), sino que también genera más residuos y desechos (ej. nutrientes y plaguicidas, antibióticos, aguas residuales) que se concentran peligrosamente en zonas puntuales, lo cual afecta negativamente al ambiente (FAO, 2007; Viglizzo, 2008).

\section{Figura $N^{\circ} 5$}

Resultados de los indicadores: a) superficie ganadera, b) cantidad de cabezas de ganado bovino, c) intensidad ganadera en los dos períodos (M1 y M2) para los grupos de partidos (AGR; GAN)
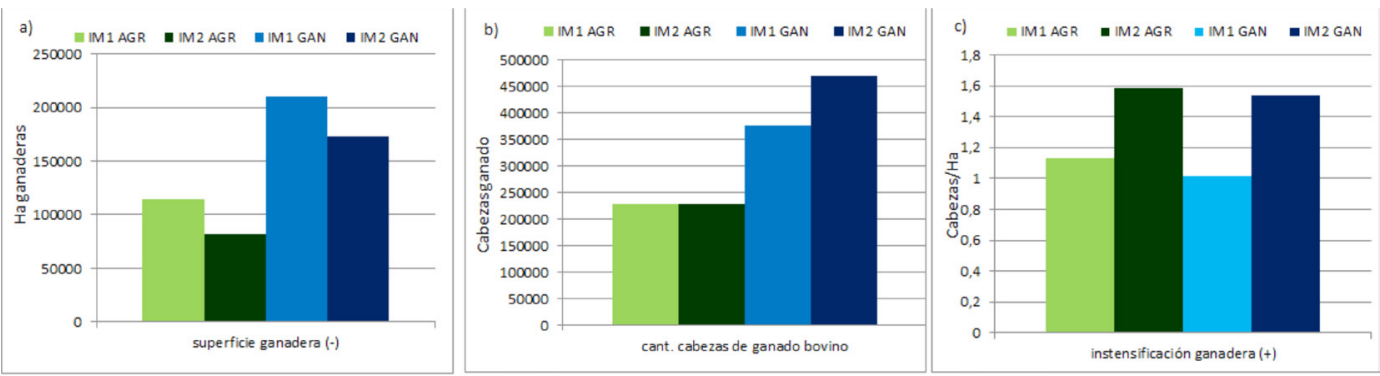

Fuente: Elaboración propia.

\section{Dimensión económica-productiva}

Confirmando la predicción planteada, los resultados muestran que los partidos ganaderos presentaron un mayor impacto en todos los indicadores seleccionados dentro de esta dimensión (Figura $\mathrm{N}^{\circ} 2$ ). El uso de insumos químicos (en litros) tuvo un aumento importante en ambos grupos de partidos, siendo mayor en los ganaderos (370\%) por efecto principalmente del incremento en la superficie sembrada con soja, cultivo que requiere de mayor cantidad de dichos insumos (Mosciaro et al., 2011). Aun así, el valor absoluto fue considerablemente mayor en los partidos agrícolas (Figura $\mathrm{N}^{\circ}$ 6). Resultados similares surgen de la cantidad empleada de insumos químicos (en $\mathrm{kg}$ ), si bien las diferencias son menores entre grupos de partidos. En este caso, la disminución del maíz y trigo, cultivos que requieren una mayor cantidad de estos insumos, no compensó el aumento en el resto de cultivos. La mayor utilización de insumos sería un aspecto positivo desde el punto de vista del desarrollo económico en el caso que la compra de dichos materiales se realice localmente, aunque no necesariamente se da esta situación, además de no contar el costo ambiental y social. Estos resultados podrían estar asociados al mayor aumento en la dependencia económica respecto de la actividad agropecuaria en los partidos ganaderos, donde esta actividad pasó a contribuir alrededor del 30\% del PBG total (el mayor impacto fue en Ayacucho, cuyo valor fue 40\%), a diferencia de los partidos agrícolas donde representó aproximadamente el $15 \%$ (con los extremos de General Pueyrredon y Balcarce, con el $2 \%$ y el $29 \%$, respectivamente) (Figura № 6). Esta situación pudo tener incidencia en la mayor concentración productiva, ya que en todos 
los partidos, salvo General Pueyrredon, la actividad agropecuaria estuvo dentro de las tres actividades que más contribuyeron al PBG de cada partido. También el hecho que el sector transporte, almacenamiento y comunicaciones haya pasado a estar en varios partidos dentro de las tres actividades principales puede ser reflejo de la mayor concentración y dependencia de la actividad agropecuaria en estos partidos, si bien este sector también incluye el transporte de personas o las comunicaciones, que no necesariamente están relacionadas a dicha actividad.

Estos resultados pueden ser negativos desde el punto de vista de la vulnerabilidad económica, principalmente por la menor diversificación y la especialización en determinada actividad -y en algunos casos, cultivo- la cual puede resultar muy afectada por la inestabilidad del clima, las plagas y las variaciones del mercado, sumado a la dependencia de determinados insumos. Además de que todo ello repercute en el bienestar para la población local (Teubal, 2009). El cambio podría ser positivo para los partidos más beneficiados en este aspecto si el aumento en la actividad agropecuaria se amplía en los distintos eslabones de la cadena agroindustrial. El mayor empleo generado en éstos podría compensar (al menos en parte, dado que la mayor eficiencia y utilización de tecnología hace que se requiera menor mano de obra en general) la pérdida de empleo rural generada por la mayor mecanización y la disminución de la actividad ganadera, la cual emplea el doble de personas que la agricultura tradicional (Viglizzo, 2008, Neiman, 2010). Si bien el precio de la tierra mostró un aumento mayor en los partidos ganaderos, en términos absolutos sigue siendo considerablemente más alto en los partidos agrícolas.

Figura $\mathrm{N}^{\circ} 6$

Resultados de los indicadores: a) uso de insumos químicos para la producción agrícola en miles de litros y b) en miles de kilogramos, c) concentración de las 3 actividades productivas principales,

d) dependencia de la actividad agropecuaria en los dos períodos (M1 y M2) para los grupos de partidos (AGR; GAN)
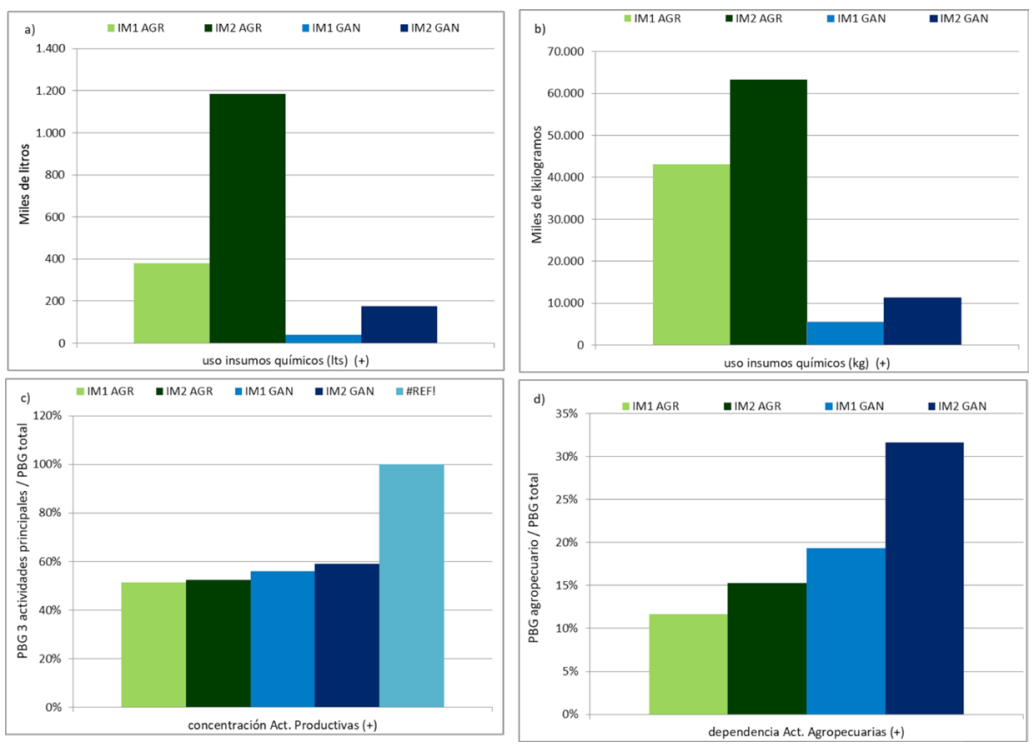

Fuente: Elaboración propia. 


\section{Dimensión socio-demográfica}

Dentro de esta dimensión, los resultados confirmaron la predicción enunciada, mostrando los partidos agrícolas un mayor impacto en todos los indicadores, salvo en la población urbana (Figura $\mathrm{N}^{\circ} 2$ 2). Los resultados evidencian disminución de la cantidad de EAPs totales y en mayor medida de las de menos de 200ha. en ambos grupos de partidos, siendo más pronunciado en los agrícolas (Figura $N^{\circ} 7$ ), principalmente en Balcarce, donde el valor disminuyó un $59 \%$ y un $72 \%$ respectivamente. Tandil también tuvo una importante reducción en estas dos variables, pudiendo relacionarse con que estos dos partidos evidenciaron con fuerza el desplazamiento de la ganadería hacia otras zonas o su intensificación. El aumento en el tamaño medio de las EAPs de más de 500ha también fue mayor en este grupo de partidos, principalmente en General Pueyrredon, donde aumentó un $50 \%$, mientras que en Tandil casi no hubo variación. Estos resultados muestran una tendencia general de las estructuras agrarias capitalistas donde se manifiesta una reducción de las producciones familiares (Mikkelsen, 2008), siendo el caso de Balcarce, donde las EAPs de menor tamaño tienden a ser alquiladas en su totalidad para producir cultivos anuales (Urcola et al., 2015). Por otro lado, Ayacucho y Mar Chiquita fueron los partidos que menos redujeron la cantidad total y de las pequeñas EAPs, siendo Ayacucho uno de los que menos redujo la superficie ganadera. En la Cuenca Mar Chiquita, Zelaya et al. (2016) encontraron que en el período 1999-2013 se registró un aumento en los sistemas de producción agrícolas (124\%), hortícolas (62\%) y mixtos (19\%), mientras que hubo una disminución del $41 \%$ de los sistemas ganaderos.

La reducción en la cantidad de EAPs de menor tamaño en el grupo de partidos agrícolas y la relativa conversión hacia sistemas agrícolas puros puede derivar en una pérdida de los beneficios asociados a una mayor diversificación de las EAPs, como una reducción de los riesgos económicos y un aumento de la productividad, estabilidad y sustentabilidad de los sistemas productivos, sumado a la conservación de los recursos naturales, principalmente el suelo (Requesens y Silva, 2011). Por su parte, la menor afectación en la cantidad y tamaño de las EAPs en los partidos ganaderos, sumado a que estos partidos aún conservan aproximadamente el $70 \%$ de tierras destinadas a este uso y que, si bien disminuyeron los sistemas de producción ganaderos puros, la conversión fue principalmente hacia sistemas mixtos (Zelaya et al., 2016), puede considerarse un resultado positivo desde diferentes perspectivas: de la diversificación productiva (incluyendo el hecho de que permite mantener en parte la infraestructura necesaria para continuar la actividad), como desde la perspectiva social (mayor demanda de mano de obra) y cultural (mantenimiento de conocimientos y tradiciones locales), pudiendo además otorgar resiliencia al sistema ante un escenario de cambio en las retenciones vinculadas a la exportación o en los precios de los productos agropecuarios.

En cuanto a los cambios demográficos (Figura $N^{\circ} 7$ ), se vincula con lo explicado precedentemente, donde la reducción en la actividad ganadera y en el número de EAPs aparece asociada con un incremento de la superficie que es vendida o rentada por los pequeños productores, los cuales en consecuencia modifican sus lugares de residencia definiendo un traslado territorial hacia pequeñas localidades o ciudades próximas. Para referir a dos situaciones concretas, Tandil y General Pueyrredon mostraron una disminución importante en la población rural dispersa, la cual fue más pronunciada en los partidos agrícolas, donde la reducción fue de aproximadamente $50 \%$. El aumento en la utilización de tecnología, asociada a la sustitución de la mano de obra local (principal sostén de las familias rurales de la zona y motor de los pueblos rurales) por maquinaria e insumos, sumado a otros factores directos e indirectos (ej. mejores oportunidades sociales), contribuye 
Figura $\mathrm{N}^{\circ} 7$

Resultados de los indicadores: a) cantidad total de EAPS, b) cantidad de EAPs < $200 \mathrm{Ha}, \mathrm{c}$ ) tamaño medio de las EAPs > 500Ha y d) población rural dispersa, en los dos períodos considerados (M1 y M2) para los dos grupos de partidos (AGR; GAN)
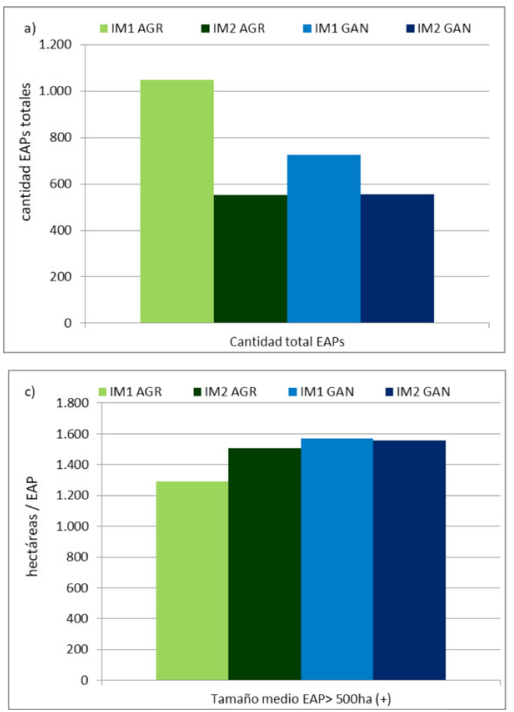
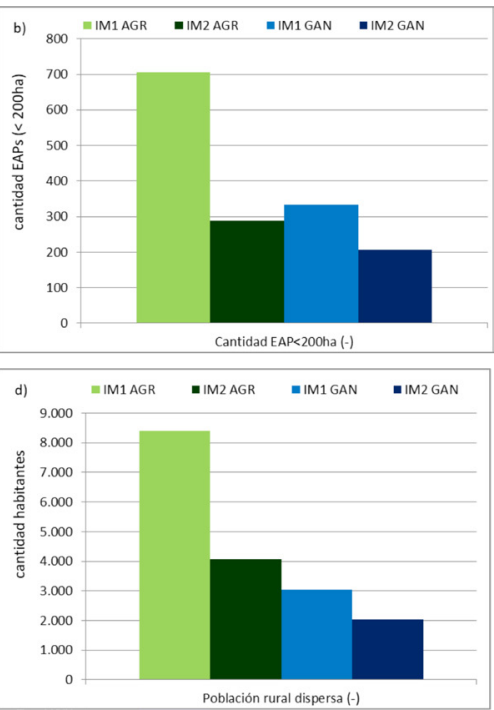

Fuente: Elaboración propia.

a la pérdida de la capacidad de los territorios para sostener una población rural estable (Viglizzo, 2008). Un caso "aparte" sería General Pueyrredon, ya que presenta la mayor disminución de población rural, aun cuando, dentro de los partidos agrícolas, fue el que menos disminución de superficie ganadera y de EAPs tuvo; esto se explicaría desde la reclasificación censal de lo que es definido como población rural (Ares y Mikkelsen, 2013) y por su menor dependencia económica de la actividad agropecuaria.

\section{Índice del proceso de agriculturización}

Al integrar las variaciones en los indicadores considerados para cada una de las dimensiones con el signo de cambio esperado y el factor de importancia en el Índice del Proceso de Agriculturización (IPA), se encontró que los partidos ganaderos tuvieron un IPA total levemente mayor que los partidos agrícolas (Figura $\mathrm{N}^{\circ}$ 8). Un resultado similar se obtuvo en la dimensión ecológica y, con una diferencia aún mayor, en la económico-productiva, mientras que en la dimensión socio-demográfica se dio el resultado inverso. Considerando las tres dimensiones, los partidos más afectados por el PA fueron Ayacucho y Mar Chiquita, seguido de Balcarce, General Madariaga y Tandil con resultados similares, siendo el menos impactado General Pueyrredon. En la dimensión ecológica, Tandil fue el más afectado, seguido de Ayacucho, mientras que Balcarce fue el menos perjudicado. En la dimensión económico-productiva Ayacucho y Mar Chiquita tuvieron el mayor impacto, con una diferencia importante respecto al que menor efecto tuvo, que fue General Pueyrredon (por el menor peso relativo de la agricultura en la economía de este partido). En la dimensión socio-demográfica, este último partido fue el que mostró mayores cambios (aunque por lo antes expuesto es probable que no se deban al PA), seguido de Balcarce, con una diferencia 
importante respecto de Mar Chiquita que fue el menos impactado, cuyo resultado es similar al resto de partidos ganaderos.

Balcarce y Tandil, partidos de tradición agrícola, habrían tenido menor capacidad de ampliar la superficie destinada a esta actividad, por lo que tuvieron principalmente una sustitución de trigo a soja. En Tandil se visualizó una mayor intensificación ganadera, además que disminuyó la superficie destinada a esta actividad, prácticamente no se redujo la cantidad de cabezas de ganado bovino, mientras que en Balcarce se contrajo un 13\%, pudiendo ser la explicación a la mayor disminución en la cantidad de EAPs, fundamentalmente las de menos de 200ha (-72\%). Sin embargo, el impacto en el despoblamiento rural afectó de manera similar a estos dos partidos, siendo el de mayor afectación General Pueyrredon. Este partido ha sido el de menor impacto del PA pudiendo asociarse a su diversificación productiva, siendo uno de los principales destinos turísticos locales, regionales y nacionales y teniendo uno de los cinturones hortícolas más importantes del país. Fue el partido con mayor aumento en el tamaño medio de las EAPs de 500has., siendo junto con Ayacucho el que alcanzó mayor incremento en la superficie con soja. Respecto de los partidos ganaderos, Mar Chiquita y Ayacucho tuvieron un patrón similar en cuanto al incremento en la superficie con soja, la intensificación ganadera y la concentración en las actividades productivas y el aumento en el precio de la tierra. Por su parte, General Madariaga tuvo mayor impacto en la superficie sembrada en general, no tanto en soja, teniendo mayor aumento en el uso de insumos químicos, junto con Ayacucho, derivado principalmente del aumento en soja y girasol, no compensando en la disminución en el resto de cultivos (ej. maíz en Madariaga y trigo en Ayacucho).

Figura $\mathrm{N}^{\circ} 8$

Índice del Proceso de agriculturización (IPA) total y por dimensiones, según partidos y agrupados en agrícolas $(A)$ o ganaderos $(G)$

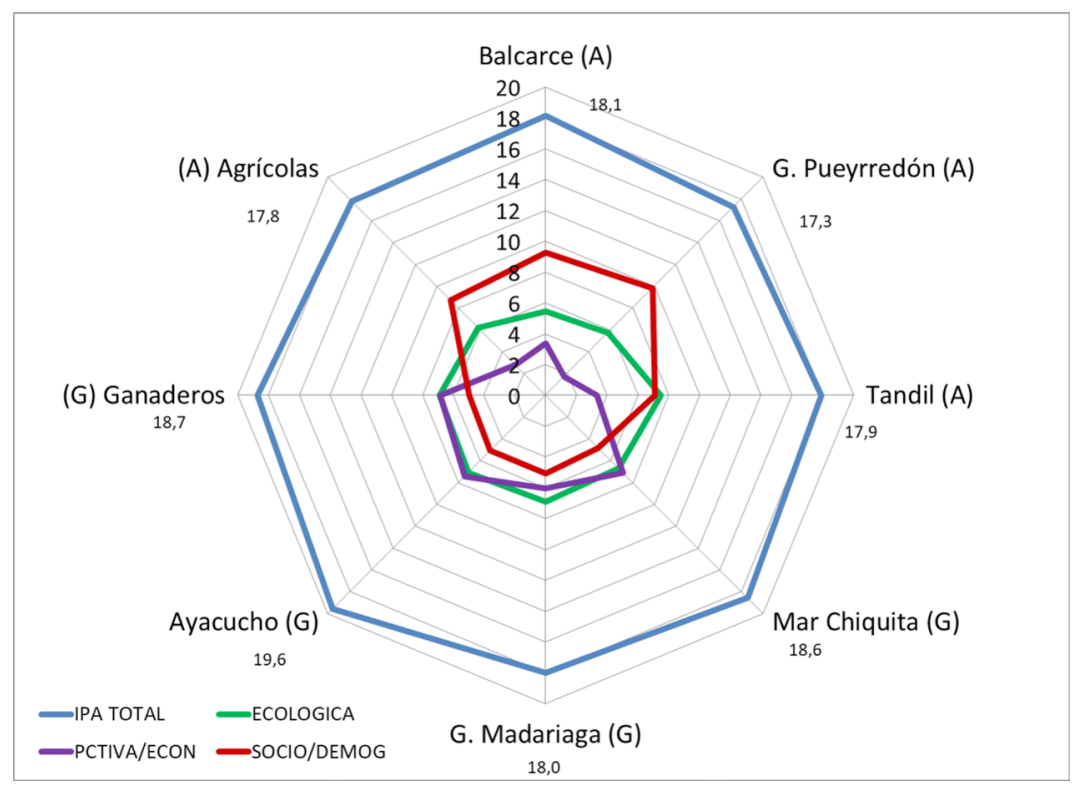

El valor del IPA total está expresado debajo de la referencia.

Fuente: Elaboración propia. 
La integración de los indicadores según las diferentes dimensiones analizadas fue un aporte metodológico cuyo resultado también es importante, dado que el desarrollo de las ciencias sociales y ecológicas de manera independiente ha dificultado comprender la complejidad que caracteriza a los socio-ecosistemas y llevado a que las recomendaciones propuestas fallen (Ostrom, 2009). El alcance global y difuso, que compromete al territorio desde lo ambiental, social, económico y político-institucional, afecta al bienestar de la población (Manzanal, 2017). Se debe considerar que las diferentes variables socio-económicas y ecológicas pueden tener una respuesta relativamente rápida (ej. niveles de humedad en suelo) o pueden ser de dinámica más lenta (e.g. estructura demográfica poblacional) (Chapin et al., 2009) afectando de diferente manera al PA y la forma de abordarlo. Por ello, es importante considerar todas las variables, principalmente aquellas que son de respuesta más lenta, porque los cambios pueden observarse una vez pasado el umbral crítico donde la pérdida es irreversible. Por ejemplo, en el caso de los saberes, costumbres y tradiciones de la comunidad, dado que el cambio de una agricultura basada en una tecnología de procesos a una basada en insumos implica la pérdida de muchos de estos valores (Giordano y Marasas, 2008).

Las estrategias para avanzar en el desarrollo sustentable del territorio están condicionadas por los factores culturales, institucionales, ambientales y sociales (Forero et al., 2014). Las mismas estarán mejor enfocadas y tendrán mayor compromiso en su cumplimiento a largo plazo si se generan desde la acción conjunta de los académicos, empresarios, gobernantes y la comunidad en general, es decir con la participación de los propios actores involucrados en los procesos territoriales. El Estado debe orientar sus políticas agropecuarias hacia una agricultura más sustentable, que alimente y de trabajo a su población, siguiendo los propios intereses nacionales (Mikkelsen,

Figura $\mathrm{N}^{\circ} 9$

El Proceso de agriculturización impacta en las distintas dimensiones generando mayor vulnerabilidad -ecológica, económica, social- siendo necesario integrar a la dimensión político-institucional para lograr un desarrollo sustentable del territorio

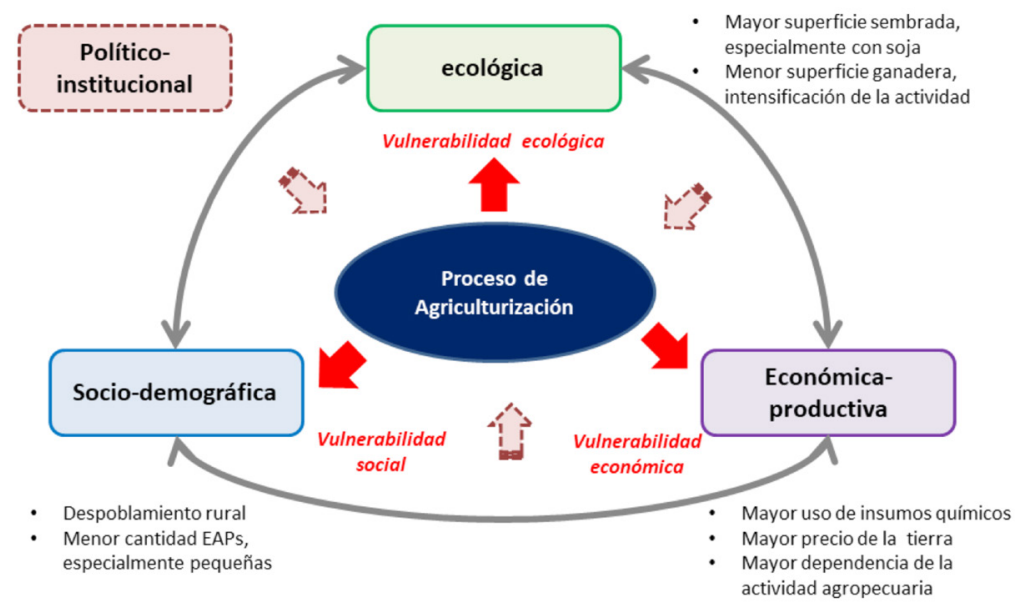

Fuente: Elaboración propia. 
2008). Si se quiere que los productores, quienes buscan obtener una producción rentable de sus campos, realicen prácticas más sustentables (ej. reducir las pulverizaciones o mantener bordes con vegetación natural), es necesario mostrarles los beneficios ambientales, económicos y sociales de estas prácticas (van der Meulen et al., 1996). Dado que el proceso de agriculturización impacta en la dimensión ecológica, económico-productiva y socio-demográfica, generando vulnerabilidad en el territorio -mayor cuanto mayor sea el impacto de este proceso - se hace necesario integrar la dimensión político-institucional con el resto de dimensiones -y sus interrelacionespara lograr un desarrollo sustentable del territorio (Figura $N^{\circ} 9$ ).

\section{Consideraciones finales}

A nivel mundial se estima que la demanda de granos se duplicará para el 2050 (Tilman et al., 2002), existen fuertes indicios de que la tendencia de la agricultura actual continuará en los próximos años (Manzanal, 2017). Por ello, resulta relevante analizar estos procesos territoriales de manera holística (Paruelo et al., 2006; Manuel-Navarrete y Gallopín, 2007), considerando sus consecuencias directas e indirectas y sus efectos a corto y largo plazo, siendo este trabajo un aporte en este sentido. Las implicancias de los cambios registrados en las diferentes dimensiones estudiadas varían para los distintos partidos analizados. Si bien todos ellos fueron examinados desde la perspectiva del proceso de agriculturización, algunos cambios económicos pueden considerarse positivos en términos de desarrollo local (ej. aumento de la productividad), mientras que los impactos ecológicos y sociales resultan en general negativos desde la perspectiva de la sustentabilidad ambiental y social de tal desarrollo. Los resultados encontrados resultan relevantes de cara a los diferentes escenarios que puedan producirse en los próximos años, considerando que la agriculturización estaría actualmente en una etapa de desaceleración o cambio (particularmente en términos de la contribución relativa de la soja). Frente a un escenario en el que los precios de los commodities continúen bajando y la demanda de carne vuelva a posicionarse, se podría pensar que la región ha perdido parte de su capacidad productiva de carne al convertir masivamente tierras para la agricultura y perder infraestructura y mano de obra rural. Por otra parte, los partidos más agrícolas podrían beneficiarse frente a un escenario en el que cambios en el esquema de retenciones o en los precios internacionales beneficien a la exportación de commodities diferentes a la soja, promoviendo esto una mayor diversidad en la producción. Aun así, dado que se continuaría bajo el mismo modelo de desarrollo, sus aportes a la mejora de la sustentabilidad serían limitados, principalmente desde la dimensión socio-demográfica del proceso. Estos resultados pueden contribuir a guiar a los tomadores de decisiones hacia la implementación de políticas de desarrollo basadas en un enfoque sustentable y a identificar y resolver los conflictos entre diferentes actores y dimensiones de la sustentabilidad (Valentin y Spangenberg, 2000; Reed et al., 2005).

\section{Referencias bibliográficas}

ADÁMOLI, J. Problemas ambientales de la agricultura en la región chaqueña. En: BROWN, A.; MARTINEZ ORTIZ, U.; ACERBI, M. y CORCUERA, J. (editores). La situación Ambiental Argentina 2005. Buenos Aires: Fundación Vida Silvestre, 2006. p. 436-441. 
AIZEN, M. A.; GARIBALDI, L.A. y DONDO M. Expansión de la soja y diversidad de la agricultura argentina. Ecología Austral, 2009, N¹9, p.45-54.

ANGELINI, H. Informe técnico sobre clasificaciones de cobertura del suelo en el área de influencia de la EEA INTA Balcarce, 2017. (inédito).

APARICIO, V.; DE GERÓNIMO, E.; HERNÁNDEZ GUIJARRO, K.; PÉREZ, D.; PORTOCARRERO, R. y VIDAL, C. Los plaguicidas agregados al suelo y su destino en el ambiente. Buenos Aires: Ediciones INTA, 2015.

ARES, S. y MIKKELSEN, C. Más allá de Mar del Plata. Dinámica sociodemográfica en las localidades menores del Partido de General Pueyrredon. En: Memoria de la Jornada Territorio y hábitat. Experiencias académicas y profesionales. Luján: Departamento de Ciencias Sociales, Universidad Nacional de Luján, 2013.

AUER, A. Servicios ecosistémicos culturales y agriculturización en el sudeste bonaerense - Cuenca de Mar Chiquita. Mar del Plata: Tesis de Doctorado, Facultad de Ciencias Agrarias, Universidad Nacional de Mar del Plata, 2017.

AUER, A.; MACEIRA, N. \& NAHUELHUAL, L. Agriculturisation and trade-offs between commodity production and cultural ecosystem services: A case study in the Argentinean Pampas. Journal of Rural Studies, 2017, N53, p. 88-101.

BARRAL, M.P. y MACEIRA N. Land-use planning based on ecosystem service assessment: a case study in the Southeast Pampas of Argentina. Agriculture, Ecosystems and Environment, 2012, No 154, p.34-43.

BARSKY, O. y GELMAN, J. Historia del Agro Argentino, desde la Conquista hasta fines del siglo XX. Buenos Aires: Grijalbo Mondadori, 2001.

BROWN, G. \& RAYMOND, C. The relationship between place attachment and landscape values: toward mapping place attachment. Applied Geography, 2007, №27, p.89-111.

CHAPIN LLL, F.S.; FOLKE, C. \& KOFINAS, G.P. A Framework for understanding change. In: Principles of ecosystem stewardship: resilience-based natural resource management in a changing world. New York: Springer, 2009, p. 3-28.

COMISIÓN INTERNACIONAL SOBRE MEDIO AMBIENTE Y DESARROLLO DE LAS NACIONES UNIDAS. Informe Brundtland, titulado "Nuestro Futuro Común". ONU, 1987.

COMPAÑIA ARGENTINA DE TIERRAS. Años 2003 y 2015. Disponible en Internet: http://www.cadetierras.com.ar/

CRAVIOTTI, C. Nuevos agentes en la producción agropecuaria, ¿nuevos sujetos del desarrollo rural? En: NEIMAN, G y CRAVIOTTI, C. (compiladores). Entre el campo y la ciudad: desafíos y estrategias de la pluriactividad en el agro. Buenos Aires: CICCUS, 2005, p. 49-67. 
DIRECCIÓN PROVINCIAL DE ESTADÍSTICA. GOBIERNO DE LA PROVINCIA DE BUENOS AIRES. Producto Bruto Geográfico. Año 1993 y 2003 por partidos. Disponible en Internet: http://www.estadistica.ec.gba.gov.ar/dpe/images/headers/pdfs/informetotalpbg.pdf

FORERO, E.L.; HERNÁNDEZ, Y.T. y ZAFRA, C.A. Percepción latinoamericana de cambio climático: metodologías, herramientas y estrategias de adaptación en comunidades locales. Una revisión. Revista U.D.C.A Actualidad \& Divulgación Científica, 2014, Vol. 17; № 1, p.73-85.

GALLOPÍN, G.C. Sostenibilidad del desarrollo en América Latina y el Caribe: cifras y tendencias Honduras. Santiago de Chile: CEPAL Naciones Unidas, 2006.

GASPARRI, I. y GRAU, R. Patrones regionales de deforestación en el subtrópico argentino y su contexto ecológico y socio-económico. En: BROWN, A.; MARTINEZ ORTIZ, U.; ACERBI, M. y CORCUERA, J. (editores). La situación ambiental Argentina 2005. Buenos Aires: Fundación Vida Silvestre, 2006, p. 442-446.

DOMÍNGUEZ, D y SABATINO, P. Con la soja al cuello: crónica de un país hambriento productor de divisas. En: ALIMONDA, H. (organizadores). Los Tormentos de la Materia. Contribuciones para una Ecología Política latinoamericana. Buenos Aires: CLACSO, 2006.

GIORDANO, G. y MARASAS, M. Tecnologías apropiadas para la agricultura familiar: análisis y reflexiones sobre su generación en situaciones representativas de la región pampeana periurbana. En: 4a Congreso Internacional de la Red SIAL. Alimentación, Agricultura Familiar y Territorio. Mar del Plata, Argentina., 27 al 31 de octubre de 2008, p. 1-14.

GRAS, C. y HERNÁNDEZ, V. Los pilares del modelo agribusiness y sus estilos empresariales. En: GRAS, C. y HERNÁNDEZ, V. (compiladores) El Agro como negocio. Producción, Sociedad y Territorios en la Globalización. Buenos Aires: Editorial Biblos, 2013, p. 17-46.

GRAS, C. y HERNÁNDEZ, V. Radiografía del nuevo campo argentino: del terrateniente al empresario transnacional. Buenos Aires: Siglo Veintiuno, 2016, p. 115-136.

HERRERA, L.; LATERRA, P.; MACEIRA, N.; MARTíNEZ, G. \& ZELAYA, K. Fragmentation status of tussock grasslands relicts in flooding Pampas, Argentina. Rangeland Ecology \& Management, 2009, $N^{\circ} 62, p .73-82$.

INSTITUTO NACIONAL DE ESTADISTICA Y CENSOS (INDEC). Censo nacional agropecuario (CNA) año 1988 y 2002. Disponible en Internet: http://www.indec.gob.ar/cna_index.asp

INSTITUTO NACIONAL DE ESTADISTICA Y CENSOS (INDEC). Censo nacional de Población y Vivienda año 1991, 2001 y 2010. Disponible en Internet:

http://www.indec.gov.ar/nivel4_default.asp?id_tema_1=2\&id_tema_2=41\&id_tema_3=135

JOBBÁGY, E.G. Servicios hídricos de los ecosistemas y su relación con el uso de la tierra en la llanura chaco-pampeana. En: LATERRA, P. JOBBÁGY, E.G. y PARUELO, J.M. (editores). Valoración de servicios ecosistémicos: conceptos, herramientas y aplicaciones para el ordenamiento territorial. Buenos Aires: Ediciones INTA, 2011, p.163-183. 
LATERRA, P.; CASTELLARINI, F. y ORÚE, E. Ecoser: un protocolo para la evaluación biofísica de servicios ecosistémicos y la integración con su valor social. En: LATERRA, P.; JOBBÁGY, E.G. y PARUELO, J.M. (editores). Valoración de servicios ecosistémicos: conceptos, herramientas y aplicaciones para el ordenamiento territorial. Buenos Aires: Ediciones INTA, 2011, p. 359-390.

LEÓN, R. Geographic limits of the region, Geomorphology and geology, Regional subdivisions, Floristic aspects, Description of the vegetation. In: COUPLAND, R.T. (editor). Natural Grasslands: Introduction and Western Hemisphere. Ámsterdam: Elsevier, 1991, p. 369-387.

LIBERALI, A.M. Argentina: agriculturización con énfasis en la sojización. En: Geografías de la agricultura industrial sojera en la Argentina. ¿Viaje de Ida? Buenos Aires: Serie-Publicaciones del PROEG No 9, Universidad Nacional de Luján, Departamento de Ciencias Sociales, 2010, p. 47-69.

LIMA M. L.; ZELAYA K.; LATERRA P.; MASSONE H. \& MACEIRA N. A dynamic simulation model of land cover in the Dulce Creek Basin, Argentina. In: 1ST CONFERENCE ON SPATIAL STATISTICS. Procedia Environmental Sciences, 2011, № 7, p. 194-199.

LLANOS HERNÁNDEZ, L. Espacio, región y territorio: una perspectiva teórica. En: LLANOS HERNÁNDEZ, L. (editor). Territorio y apropiación del espacio social en las tierras indias de Chiapas: rupturas y continuidades en los procesos de cambio social. Madrid: Plaza y Valdés, 2013, p. 27-45.

MANUEL-NAVARRETE, D. y GALLOPÍN, G. Integración de políticas, sostenibilidad y agriculturización en la pampa argentina y áreas extrapampeanas. Santiago de Chile: CEPAL Naciones Unidas, Seminarios y Conferencias No 50, 2007, p.1-34.

MANZANAL, M. Territorio, Poder y Sojización en el Cono Sur latinoamericano. El caso argentino. Mundo Agrario, 2017, Vol. 18, N 37, p. 1-26.

MINISTERIO DE AGRICULTURA GANADERIA Y PESCA (MAGYP). Sistema Integrado de Información Agropecuaria (SIIA). Disponible en Internet:

https://datos.magyp.gob.ar/reportes.php?reporte=Estimaciones

MIKKELSEN, C.A. La expansión de la soja y su relación con la agricultura industrial. Revista Universitaria de Geografía, 2008, Vol. 17, p. 165-188.

MORELLO, J.; RODRÍGUEZ, A.F. y PENGUE, W. Mirando al revés: la ciudad desde el campo: el caso de la llanura chaco-pampeana argentina. En: BROWN, A.; MARTINEZ ORTIZ, U.; ACERBI, M. y CORCUERA, J. (editores). La situación ambiental Argentina 2005. Buenos Aires: Fundación Vida Silvestre, 2006, p. 447-455.

MOSCIARO, M.; NATINSON, P. y TOSI, J.C. Análisis de la situación actual y de la sustentabilidad económica de sistemas característicos de los territorios del CERBAS. Buenos Aires: INTA, Proyecto Regional BASUR-720071 y Proyecto Específico AEES - 302442, 2011.

MOSCUZZA, C. H. Intensificación de la producción agropecuaria. En: FERNÁNDEZ CIRELLI, A.; MOSCUZZA, C.H.; PÉREZ CARRERA, A.L. y VOLPEDO, A.V. (editores). Aspectos ambientales de las actividades agropecuarias. Buenos Aires: AgroVet, 2010, p. 43-56. 
NEIMAN, G. Los estudios sobre el trabajo agrario en la última década. Una revisión para el caso argentino. Mundo agrario, 2010, Vol. 10, N²0, p.1-20.

ORGANIZACIÓN DE LAS NACIONES UNIDAS PARA LA AGRICULTURA Y LA ALIMENTACIÓN (FAO). El estado mundial de la agricultura y la alimentación. Parte 1: Pagos a los agricultores por servicios ambientales. Roma: IT Colección FAO: Agricultura, №38, 2007, p. 2-128.

OSTROM, E. A general framework for analyzing sustainability of socio-ecological systems. Science, 2009, N³25, p. 419-422.

PARUELO, J.M.; GUERSCHMAN, J.P.; PIÑEIRO, G.; JOBBÁGY, E.G.; VERÓN, S.R.; BALDI, G. y BAEZA, $S$. Cambios en el uso de la tierra en Argentina y Uruguay: Marcos conceptuales para su análisis. Agrociencia, 2006, Vol. X, № 2, p. $47-61$.

PORTO-GONÇALVES, C.W. El desafío ambiental. Programa de las Naciones Unidas para el Medio Ambiente. México D.F., 2006.

QUIRÓS, R.; RENNELLA, A.; BOVERI, M.; ROSSO, J.J. y SOSNOVSKY, A. Factores que afectan la estructura y el funcionamiento de las lagunas pampeanas. Ecología Austral, 2002, № 12, p. 175-185.

REBORATTI, C. La Argentina rural entre la modernización y la exclusión. In: GERAIGES DE LEMOS, A.M.; ARROYO, M. \& SILVEIRA, M.L. (editores). América Latina: Cidade, campo e turismo. Buenos Aires: CLACSO, 2006, p. $175-187$.

REED, M.S.; FRASER, E.D.G. \& DOUGILL, A.J. An adaptive learning process for developing and applying sustainability indicators with local communities. Ecological Economics, 2005, N 59, p.406418.

REQUESENS, E. y SILVA, L. Tendencias en el uso de la tierra y diversidad productiva en establecimientos agropecuarios del centro-sur de la provincia de Buenos Aires. Agriscientia, 2011, $\mathrm{N}^{\circ}$ XXVIII, p. 75-83.

SABATINO, M.; MACEIRA, N. y AIZEN, M.A. Direct effects of habitat area on interaction diversity in pollination webs. Ecological Applications, 2010, Vol. 20, № 6, p. 1491-1497.

SECRETARÍA DE AMBIENTE Y DESARROLLO SUSTENTABLE DE LA NACIÓN (SAyDS). Sistema de indicadores de desarrollo sostenible. Buenos Aires: SAyDS, 2009.

SILI, M. La Argentina rural: de la crisis de la modernización agraria a la construcción de un nuevo paradigma de desarrollo de los territorios rurales. Buenos Aires: Ediciones INTA, 2005.

TAPELLA, E. Heterogeneidad social y valoración diferencial de servicios ecosistémicos: un abordaje multi-actoral en el oeste de Córdoba (Argentina). Córdoba: Tesis de Doctorado, Facultad de Ciencias Agropecuarias, Universidad Nacional de Córdoba, 2011, p. 115-254. 
TEUBAL, M. Expansión de la Soja Transgénica en la Argentina. En: PEREZ, M. (editor). Promesas y peligros de la liberalización del comercio agrícola: lecciones desde América Latina. La Paz: 2009. p. $73-90$

TILMAN, D.; CASSMAN, K.G.; MATSON, P.A.; NAYLOR, R. \& POLASKY, S. Agricultural sustainability and intensive production practices. Nature, 2002, N 418, p. 671-677.

URCOLA, H.A.; DE SARTRE, X.A.; VEIGA JR.I.; ELVERDÍN, J. \& ALBALADEJO, C. Land tenancy, soybean, actors and transformations in the pampas: a district balance. Journal of Rural Studies, 2015, No 39, p.32-40.

VALENTIN, A. \& SPANGENBERG, J.H. A guide to community sustainability indicators. Environmental Impact Assessment Review, 2000, № 20, p. 381-392.

VAN DER MEULEN, H.A.B.; DE SNOO, G.R. \& WOSSINK, G.A.A. Farmers' perception of unsprayed crop edges in the Netherlands. Journal of Environmental Management, 1996, N 47, p. 241-255.

VIGLIZZO, E.F. Agricultura, clima y ambiente en Argentina: tendencias, interacciones e impactos. En: SOLBRIG, O.T. y ADÁMOLI, J. (editores). Agro y ambiente: una agenda compartida para el desarrollo sustentable. Buenos Aires: Foro de la Cadena Agroindustrial Argentina, 2008, p. 1-25.

ZARRILLI, A. ¿Una agriculturización insostenible? La provincia del Chaco, Argentina (1980-2008). Historia Agraria, 2010, № 51, p. 143-176.

ZELAYA, K. Descripción y modelización de la dinámica del uso de la tierra en la cuenca hidrológica de Mar Chiquita. Mar del Plata: Tesis de Magister Scientiae, Facultad de Ciencias Agrarias, Universidad Nacional de Mar del Plata, 2011.

ZELAYA, K.; VAN VLIET, J. \& VERBURG, P.H. Characterization and analysis of farm system changes in the Mar Chiquita basin, Argentina. Applied Geography, 2016, № 68, p. 95-103. 
